\title{
Lewis Base Activation of Lewis Acids. 2. Addition of Silyl Ketene Acetals to Aldehydes
}

\author{
Scott E. Denmark*, Thomas Wynn, Gregory L. Beutner \\ Roger Adams Laboratory, Department of Chemistry, University of Illinois \\ Urbana, Illinois 61801
}

\section{SUPPORTING INFORMATION}

\section{General Experimental}

All reactions were performed in oven-dried $\left(140^{\circ} \mathrm{C}\right)$ or flame-dried glassware under an atmosphere of dry $\mathrm{N}_{2}$. The following reaction solvents were distilled from the indicated drying agents: dichloromethane $\left(\mathrm{P}_{2} \mathrm{O}_{5}\right)$, benzene (Fisher ACS grade), hexanes (Fisher ACS grade), tetrahydrofuran ( $\mathrm{Na}$, benzophenone) and diethyl ether (Na, benzophenone). Solvents for chromatography and filtration were technical grade and distilled from the indicated drying agents: hexane $\left(\mathrm{CaCl}_{2}\right)$, ethyl acetate $\left(\mathrm{K}_{2} \mathrm{CO}_{3}\right)$, dichloromethane $\left(\mathrm{CaCl}_{2}\right)$. Column chromatography was performed using EM Science 230-400-mesh silica gel. Benzaldehyde (2a), 1-napthaldehyde (2b), p-tolualdehyde (2d), p-anisaldehyde (2e), p-trifluoromethylbenzaldehyde (2f), trans-cinnamaldehyde (2g), $\alpha$-methyl-trans-cinnamaldehyde $\quad(\mathbf{2 h}), \quad$ furfural $\quad(\mathbf{2 i})$, cyclohexanecarboxaldehyde $(\mathbf{2} \mathbf{j})$ and hydrocinnamaldehyde (2k) were freshly distilled before use. 2-Naphthaldehyde (2c) (Aldrich) was sublimed prior to use. 1-(tertButyldimethylsilyloxy)-1-methoxyethene (1) (Aldrich) was stored over $3 \AA$ molecular sieves. Silicon tetrachloride (Aldrich) was heated at reflux for $24 \mathrm{~h}$ and then distilled prior to use. HMPA was distilled and then stored over $3 \AA$ molecular sieves prior to use. tert-Butyl dimethylsilyl chloride (TBSCl, Aldrich) and 3,5-dintrobenzoyl chloride (Aldrich) were used without further purification. Methyl propanoate, ethyl propanoate and tert-butyl propanoate (Aldrich) were stored over $3 \AA$ molecular sieves. Propanoic acid phenyl ester (TCI America) was stored over $3 \AA$ molecular sieves. Diisopropyl amine, pyridine and hexamethyldisilazane were freshly distilled from $\mathrm{CaH}_{2}$ prior to use. Tetrabutylammonium iodide was recrystallized from 
toluene and dried under vacuum prior to use. All reaction temperatures correspond to internal temperatures measured by Teflon-coated thermocouples unless otherwise noted.

${ }^{1} \mathrm{H}$ NMR, ${ }^{13} \mathrm{C} \mathrm{NMR},{ }^{19} \mathrm{~F}$ NMR, and low temperature spectra were recorded on a Varian Unity $400\left(400 \mathrm{MHz},{ }^{1} \mathrm{H} ; 100 \mathrm{MHz},{ }^{13} \mathrm{C}, 376 \mathrm{MHz}{ }^{19} \mathrm{~F}\right)$, Unity $500\left(500 \mathrm{MHz},{ }^{1} \mathrm{H} ; 126 \mathrm{MHz},{ }^{13} \mathrm{C}\right.$, $202 \mathrm{MHz}$ ) spectrometers. Spectra were referenced to residual chloroform (7.26 ppm, ${ }^{1} \mathrm{H}, 77.23$ ppm, ${ }^{13} \mathrm{C}$ ), ${ }^{19} \mathrm{~F}$ spectra were referenced externally to $\mathrm{C}_{6} \mathrm{~F}_{6}$. Chemical shifts are reported in ppm, multiplicities are indicated by s (singlet), d (doublet), t (triplet), q (quartet), p (pentet) h (hextet) m (multiplet) and br (broad). Coupling constants, $J$, are reported in Hertz. Mass spectroscopy was performed by the University of Illinois Mass Spectrometer Center. EI mass spectra were performed on a 70-VSE spectrometer. FAB mass spectra were performed on a FinniganMATCH-5 spectrometer. Data are reported in the form of $(\mathrm{m} / \mathrm{z})$. Infrared spectra (IR) were recorded on a Mattson Galaxy 5020 spectrophotometer in $\mathrm{NaCl}$ cells. Peaks are reported in $\mathrm{cm}^{-1}$ with indicated relative intensities: s (strong, 67-100\%); m (medium, 34-66\%); w (weak, 0-33\%). Elemental analyses were performed by the University of Illinois Microanalytical Service Laboratory. Melting points ( $\mathrm{mp}$ ) were determined on a Thomas-Hoover capillary melting point apparatus in sealed tubes and are uncorrected. Kugelrohr distillations were performed on a Büchi GKR-50 Kugelrohr and temperatures reported are air bath temperatures. Analytical thinlayer chromatography was performed on Merck silica gel plates with QF-254 indicator. Visualization was accomplished with $\mathrm{UV}(254)$, potassium permanganate $\left(\mathrm{KMnO}_{4}\right)$ and phosphomolybdic Acid (PMA).

Optical rotation data was obtained on a JASCO DIP-360 digital polarimeter and are reported as follows: concentration $(\mathrm{c}=\mathrm{g} / 100 \mathrm{~mL})$, and solvent. Analytical supercritical fluid chromatography (SFC) was performed on a Berger Instruments packed-column SFC with builtin photometric detector $(220 \mathrm{~nm})$ using Daicel Chiralpak OD, AD, AS columns and a Regis Whelk-O1 column. Analytical capillary gas chromatography (GC) was performed on a Hewlett Packard 5890 Series II fitted with a flame ionization detector $\left(\mathrm{H}_{2}\right.$ carrier gas, $\left.16 \mathrm{~mL} / \mathrm{min}\right)$ equipped with an Astec (Chiraldex) GTA $30 \mathrm{~m}$ x $0.25 \mathrm{~mm}$ x $0.125 \mu \mathrm{m}$ column. The injector temperature was $225{ }^{\circ} \mathrm{C}$, the detector temperature was $300{ }^{\circ} \mathrm{C}$ Retention times $\left(t_{R}\right)$ and peak ratios were determined with a Hewlett Packard 3396 Series II integrator. 


\section{Literature Preparations.}

$(R, R)-N, N^{\prime}$-Bis[4,5-dihydro-3,5-dimethyl-4-(3H-dinaphtho[2,1-d:1',2'-f][1,3,2]-2-oxodiazaphosphepino)]-N,N'-dimethyl-1,5-pentanediamine $((R, R)-3)^{1}, 3$-phenyl-2-propynal $(2 \mathrm{l})^{2}$ and (E)-(1-ethoxy-propenyloxy)-tert-butyldimethylsilane $(\mathbf{5 b})^{3}$ were prepared by literature methods.

\section{Experimental Procedures.}

Representative Procedure 1. Addition of acetate-derived Silyl Ketene Acetals to Aromatic Aldehydes.

Preparation of Methyl $(R)-3-H y d r o x y-3-p h e n y l p r o p a n o a t e ~((R)-4 a)$

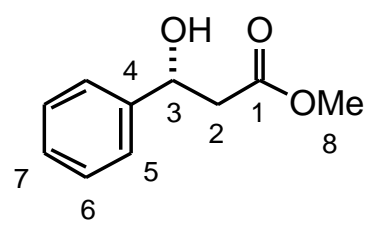

A flame-dried, 20-mL, 2-neck flask containing a solution of $84 \mathrm{mg}(0.1 \mathrm{mmol}, 0.05$ equiv) of bisphosphoramide $(R, R)-3$ in $\mathrm{CH}_{2} \mathrm{Cl}_{2}(10 \mathrm{~mL})$ was cooled to $-78{ }^{\circ} \mathrm{C}$ under nitrogen and then $200 \mu \mathrm{L}$ ( $2.0 \mathrm{mmol}, 1.0$ equiv) of benzaldehyde was added in one portion. To the resulting solution $252 \mu \mathrm{L}$ (2.2 mmol, 1.1 equiv) of $\mathrm{SiCl}_{4}$ was added and the reaction mixture was allowed to stir at $-78{ }^{\circ} \mathrm{C}$ for $5 \mathrm{~min}$. Then $523 \mu \mathrm{L}$ (2.4 mmol, 1.2 equiv) of 1-(tert-butyldimethylsilyloxy)1-methoxyethene (1) was added dropwise (over $5 \mathrm{~min}$ ) to the reaction mixture. The resulting mixture was allowed to stir at $-78{ }^{\circ} \mathrm{C}$ (bath temperature) for 15 min whereupon the cold reaction mixture was poured into a rapidly stirring solution of $1 / 1$ sat. aq. $\mathrm{KF} / 1.0 \mathrm{M} \mathrm{KH}_{2} \mathrm{PO}_{4}$ solution (50 $\mathrm{mL}$ ). This biphasic mixture was stirred vigorously for $2 \mathrm{~h}$ after which the aqueous layer was washed with $\mathrm{CH}_{2} \mathrm{Cl}_{2}(3 \times 50 \mathrm{~mL})$. The combined organic extracts were dried over $\mathrm{Na}_{2} \mathrm{SO}_{4}$, filtered and the filtrate was concentrated in vacuo. The residue was purified by column chromatography (hexanes/EtOAc, 2/1). The product-containing fractions were combined and the solvent was removed in vacuo to yield $348 \mathrm{mg}(97 \%)$ of $(R)-\mathbf{4 a}$ as a colorless oil. ${ }^{4}$ 


\section{Data for $(R)-\mathbf{4 a}$ :}

${ }^{1} \mathrm{H}$ NMR: $\quad\left(400 \mathrm{MHz}, \mathrm{CDCl}_{3}\right)$

7.40-7.34 (m, 4 H, 2 x HC(5), 2 x HC(6)), 7.31-7.27 (m, 1 H, HC(7)), 5.16 (dt, $J$ $=8.8,3.4,1 \mathrm{H}, \mathrm{HC}(3)), 3.73$ (s, $3 \mathrm{H}, \mathrm{HC}(8)), 3.23$ (bs, $1 \mathrm{H}, \mathrm{HO}), 2.75$ (dd, $J=$ 16.5, 8.8, $1 \mathrm{H}, \mathrm{H} H \mathrm{C}(2)), 2.73(\mathrm{dd}, J=16.4,6.8,1 \mathrm{H}, H \mathrm{HC}(2))$

${ }^{13} \mathrm{C} \mathrm{NMR}: \quad\left(100 \mathrm{MHz}, \mathrm{CDCl}_{3}\right)$

173.03 (C(1)), 142.63 (C(4)), 128.78 (C(6)), 128.05 (C(7)), $125.84(\mathrm{C}(5)), 70.50$ (C(3)), $52.13(\mathrm{C}(8)), 43.31(\mathrm{C}(2))$

IR: (neat)

3456 (s), 3031 (m), 3005 (w), 2953 (m), 1735 (s), 1494 (w), 1438 (s), 1403 (s), 1360(m), 1269 (m), 1200 (m), 1164 (m), 1060 (m), 1036 (m), 1025 (m), 988 (w), $762(\mathrm{~m}), 701(\mathrm{~m})$

Opt. Rot.: $\quad[\alpha]_{\mathrm{D}}^{24}+17.7(\mathrm{c}=2.05, \mathrm{EtOH})\left[\mathrm{Lit}^{2}:[\alpha]_{\mathrm{D}}^{24}(R)+12.4(\mathrm{c}=2.66, \mathrm{EtOH})\right]$

SFC: $\quad t_{R}(R)-\mathbf{4 a}, 3.81(96.3 \%) ; t_{R}(S)-\mathbf{4 a}, 4.23$ min (3.7\%) (Chiralpak AD, 200 bar, 40 ${ }^{\circ} \mathrm{C}, 3.5 \% \mathrm{CH}_{3} \mathrm{OH}$ in $\mathrm{CO}_{2}, 2.7 \mathrm{~mL} / \mathrm{min}, 220 \mathrm{~nm}$ )

\section{Preparation of Methyl $(R)-3-H y d r o x y-3-(1-n a p h t h y l) p r o p a n o a t e ~((R)-4 b)$}

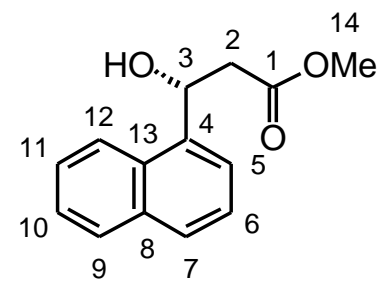

Following General Procedure 1, $271 \mu \mathrm{L}$ (2.0 mmol, 1.0 equiv) of 1-naphthaldehyde was combined with $84 \mathrm{mg}(0.1 \mathrm{mmol}, 0.05$ equiv) of bisphosphoramide $(R, R)-3,252 \mu \mathrm{L}(2.2 \mathrm{mmol}$, 1.1 equiv) of $\mathrm{SiCl}_{4}$, and $523 \mu \mathrm{L}(2.4 \mathrm{mmol}, 1.2$ equiv) of 1-(tert-butyldimethylsilyloxy)-1methoxyethene (1) to yield, after purification by column chromatography (hexanes/EtOAc, 2/1), $457 \mathrm{mg}(98 \%)$ of $(R)-\mathbf{4 b}$ as a colorless solid. An analytical sample was obtained by recrystallization from $\mathrm{Et}_{2} \mathrm{O}$ to afford colorless crystals. 


\section{Data for $\mathbf{4 b}$ :}

mp: $\quad 76-77^{\circ} \mathrm{C}\left(\mathrm{Et}_{2} \mathrm{O}\right)$

${ }^{1} \mathrm{H}$ NMR: $\quad\left(400 \mathrm{MHz}, \mathrm{CDCl}_{3}\right)$

8.05 (d, $J=8.3,1 \mathrm{H}, \mathrm{HC}(12)), 7.89$ (d, $J=7.5,1 \mathrm{H}, \mathrm{HC}(9)), 7.80$ (d, $J=8.1,1 \mathrm{H}$, $\mathrm{HC}(7)), 7.71$ (d, $J=7.0,1 \mathrm{H}, \mathrm{HC}(5)), 7.51$ (m, $3 \mathrm{H}, \mathrm{HC}(6), \mathrm{HC}(10), \mathrm{HC}(11))$, 5.93 (m, $1 \mathrm{H}, \mathrm{HC}(3)), 3.78$ (s, $3 \mathrm{H}, \mathrm{HC}(14)), 3.35$ (bs, $1 \mathrm{H}, \mathrm{HO}), 2.91$ (dd, $J=$ $16.3,5.3,1 \mathrm{H}, \mathrm{HHC}(2)), 2.90(\mathrm{dd}, J=16.3,6.0,1 \mathrm{H}, H \mathrm{HC}(2))$

${ }^{13} \mathrm{C} \mathrm{NMR}: \quad\left(100 \mathrm{MHz}, \mathrm{CDCl}_{3}\right)$

$173.31(\mathrm{C}(1)), 138.12(\mathrm{C}(4)), 133.94$ (C(8)), 130.10 (C(13)), 129.22 (C(9)), 128.52 (C(7)), 126.51 (C(11)), 125.83 (C(10)), 125.72 (C(6)), 123.11 (C(5)), $122.92(\mathrm{C}(12)), 67.50(\mathrm{C}(3)), 52.23(\mathrm{C}(14)), 42.70(\mathrm{C}(2))$

IR: (neat)

3459 (s), 3051 (s), 3007 (w), 2951 (m), 1731 (s), 1597 (w), 1511 (m), 1437 (s), 1356 (m), 1279 (m), 1206 (m), 1173 (s), 1083 (m), 1042 (s), 1001 (w), 906 (w), $861(\mathrm{w}), 803$ (s), $780(\mathrm{~s}), 737(\mathrm{w}), 695(\mathrm{w})$

MS: $\quad(E I, 70 \mathrm{eV})$ $230\left(\mathrm{M}^{+}, 31\right), 157$ (100), $129(80)$

Opt. Rot.: $\quad[\alpha]_{\mathrm{D}}^{24}+21.00(\mathrm{c}=2.12, \mathrm{EtOH})$

TLC: $\quad R_{f} 0.35$ (hexanes/EtOAc, 2/1) [UV(254)/PMA]

SFC: $\quad t_{R}(S)-\mathbf{4 b}, 5.17(9.8 \%) ; t_{R}(R)-\mathbf{4 b}, 5.91 \mathrm{~min}(90.2 \%)$ (Chiralpak OD, 200 bar, 40 ${ }^{\circ} \mathrm{C}, 9.0 \% \mathrm{CH}_{3} \mathrm{OH}$ in $\mathrm{CO}_{2}, 3.0 \mathrm{~mL} / \mathrm{min}, 220 \mathrm{~nm}$ )

Analysis: $\quad \mathrm{C}_{14} \mathrm{H}_{14} \mathrm{O}_{3}(230.26)$
Calcd:
C, 73.03;
$\mathrm{H}, 6.13 \%$
Found:
C, 72.93;
H, $6.16 \%$ 
Preparation of Methyl $(R)-3-H y d r o x y-3-(2-n a p h t h y l) p r o p a n o a t e ~((R)-4 c)$

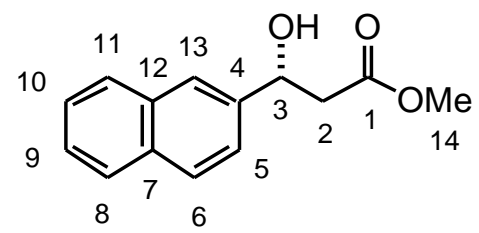

Following General Procedure 1, $312.3 \mathrm{mg}$ (2.0 mmol, 1.0 equiv) of 2-naphthaldehyde was combined with $84 \mathrm{mg}(0.1 \mathrm{mmol}, 0.05$ equiv) of bisphosphoramide $(R, R)-3,252 \mu \mathrm{L}(2.2$ mmol, 1.1 equiv) of $\mathrm{SiCl}_{4}$, and $523 \mu \mathrm{L}$ (2.4 mmol, 1.2 equiv) of 1-(tert-butyldimethylsilyloxy)-1methoxyethene (1) to yield, after purification by column chromatography (hexanes/EtOAc, 2/1), $455 \mathrm{mg}(98 \%)$ of $(R)-\mathbf{4 c}$ as a wax-like, white solid. After Kugelrohr distillation an analytically pure, colorless oil was isolated which solidified upon standing.

Data for $(R)-4 \mathrm{c}:$

bp: $\quad 145^{\circ} \mathrm{C}\left(1.8 \times 10^{-4} \mathrm{mmHg}, \mathrm{ABT}\right)$

${ }^{1} \mathrm{H}$ NMR: $\quad\left(400 \mathrm{MHz}, \mathrm{CDCl}_{3}\right)$

7.83 (m, 4 H, H(Ar)), 7.48 (m, 3 H, H(Ar)), 5.31 (m, 1 H, HC(3)), 3.74 (s, 3 H, HC(14)), 3.34 (m, $1 \mathrm{H}, \mathrm{HO}), 2.84$ (dd, $J=16.4,6.0,1 \mathrm{H}, \mathrm{H} H \mathrm{C}(2)), 2.83(\mathrm{dd}, J=$ 16.4, 2.0, $1 \mathrm{H}, \mathrm{HHC}(2))$

${ }^{13} \mathrm{C} \mathrm{NMR}: \quad\left(100 \mathrm{MHz}, \mathrm{CDCl}_{3}\right)$

$173.06(\mathrm{C}(1)), 140.03$ (C(4)), 133.48 (C(12)), 133.24 (C(7)), $128.65(\mathrm{C}(8))$, 128.25 (C(11)), 127.90 (C(5)), 126.47 (C(6)), 126.24 (C(13)), 124.68 (C(10)), 123.89 (C(9)), $70.63(\mathrm{C}(3)), 52.16(\mathrm{C}(14)), 43.28(\mathrm{C}(2))$

IR: (neat)

3442 (s), 3055 (s), 3022 (m), 2952 (m), 1732 (s), 1601 (w), 1509 (m), 1437 (s), 1404 (w), 1358 (m), 1274 (s), 1203 (m), 1169 (m), 1067 (m), 1034 (m), 897 (w), $821(\mathrm{~s}), 748(\mathrm{~s}), 692(\mathrm{w})$

MS: $\quad(\mathrm{EI}, 70 \mathrm{eV})$ $230\left(\mathrm{M}^{+} 41\right), 157$ (100), 129 (120)

Opt. Rot.: $\quad[\alpha]_{\mathrm{D}}^{24}+15.66(\mathrm{c}=2.11, \mathrm{EtOH})$

TLC: $\quad R_{f} 0.30$ (hexanes/EtOAc, 2/1) [UV(254)/PMA] 
SFC: $\quad t_{R}(R)-4 c, 4.72(96.8 \%) ; t_{R}(S)-\mathbf{4 c}, 5.26 \min (3.2 \%)$ (Chiralpak AD, 150 bar, $40{ }^{\circ} \mathrm{C}, 10.0 \% \mathrm{CH}_{3} \mathrm{OH}$ in $\mathrm{CO}_{2}, 3.0 \mathrm{~mL} / \mathrm{min}, 220 \mathrm{~nm}$ )

Analysis: $\quad \mathrm{C}_{14} \mathrm{H}_{14} \mathrm{O}_{3}(230.26)$
Calcd:
C, 73.03;
H, $6.13 \%$
Found:
$\mathrm{H}, 6.02 \%$

C, 72.82;

Preparation of Methyl $(R)-3-H y d r o x y-3-(4-m e t h y l p h e n y l) p r o p a n o a t e ~((R)-4 d)$

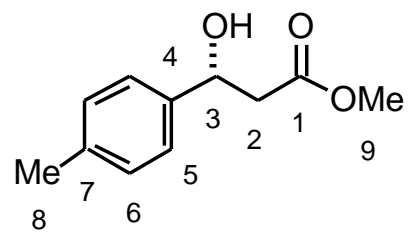

Following General Procedure 1, $236 \mu \mathrm{L}$ (2.0 mmol, 1.0 equiv) of anisaldehyde was combined with $84 \mathrm{mg}(0.1 \mathrm{mmol}, 0.05$ equiv) of bisphosphoramide $(R, R)-3,252 \mu \mathrm{L}(2.2 \mathrm{mmol}$, 1.1 equiv) of $\mathrm{SiCl}_{4}$, and $523 \mu \mathrm{L}(2.4 \mathrm{mmol}, 1.2$ equiv) of 1-(tert-butyldimethylsilyloxy)-1methoxyethene (1) to yield, after purification by column chromatography (hexanes/EtOAc, 2/1), $378 \mathrm{mg}(97 \%)$ of $(R)-\mathbf{4 d}$ as a clear oil. After Kugelrohr distillation an analytically pure, colorless oil was collected.

Data for $(R)-\mathbf{4 d}$ :

bp: $\quad 105^{\circ} \mathrm{C}\left(1.4 \times 10^{-4} \mathrm{mmHg}, \mathrm{ABT}\right)$

${ }^{1} \mathrm{H}$ NMR: $\quad\left(400 \mathrm{MHz}, \mathrm{CDCl}_{3}\right)$

$7.28(\mathrm{~d}, J=8.1,2 \mathrm{H}, \mathrm{HC}(5)), 7.18(\mathrm{~d}, J=8.1,2 \mathrm{H}, \mathrm{HC}(6)), 5.12$ (dt, $J=9.0,3.6$, $1 \mathrm{H}, \mathrm{HC}(3)), 3.75$ (s, $3 \mathrm{H}, \mathrm{HC}(9)), 3.13$, (d, $J=3.4,1 \mathrm{H}, \mathrm{HO}), 2.76$ (dd, $J=16.4$, 7.8, $1 \mathrm{H}, \mathrm{H} H \mathrm{C}(2)), 2.74(\mathrm{dd}, J=16.4,5.3,1 \mathrm{H}, H \mathrm{HC}(2)), 1.60$ (s, $3 \mathrm{H}, \mathrm{HC}(8))$

${ }^{13} \mathrm{C} \mathrm{NMR}: \quad\left(100 \mathrm{MHz}, \mathrm{CDCl}_{3}\right)$

173.08 (C(1)), 139.69 (C(4)), 137.80 (C(7)), 129.46 (C(6)), 125.80 (C(5)), 70.39 (C(3)), $52.13(\mathrm{C}(9)), 43.32(\mathrm{C}(2)), 21.34(\mathrm{C}(8))$

IR: (neat)

3450 (s), 3023 (w), 2952 (m), 2922 (m), 1737 (s), 1515 (m), 1438 (m), 1357 (w), 1267 (w), 1198 (m), 1162 (m), 1067 (w), 1035 (m), $1019(\mathrm{w}), 989(\mathrm{w}), 818(\mathrm{~m})$ 
MS: $\quad(E I, 70 \mathrm{eV})$

$194\left(\mathrm{M}^{+}, 22\right) 179$ (10), 122 (10), 121 (100), 105 (10)

Opt. Rot.: $\quad[\alpha]_{\mathrm{D}}^{24}+16.51(\mathrm{c}=2.21, \mathrm{EtOH})$

TLC: $\quad R_{f} 0.33$ (hexanes/EtOAc, 2/1) [UV(254)/PMA]

SFC: $\quad t_{R}(R)-\mathbf{4 d}, 3.97(97.2 \%) ; t_{R}(S)-\mathbf{4 d}, 4.37$ min (2.8\%) (Chiralpak AD, 150 bar, 40 ${ }^{\circ} \mathrm{C}, 4.0 \% \mathrm{CH}_{3} \mathrm{OH}$ in $\left.\mathrm{CO}_{2}, 3.0 \mathrm{~mL} / \mathrm{min}, 220 \mathrm{~nm}\right)$

Analysis: $\quad \mathrm{C}_{11} \mathrm{H}_{14} \mathrm{O}_{3}(194.23)$
Calcd:
C, 68.02;
$\mathrm{H}, 7.27 \%$

Found:

C, 67.83;

$\mathrm{H}, 7.32 \%$

Preparation of Methyl $(R)-3-H y d r o x y-3-(4-m e t h o x y p h e n y l) p r o p a n o a t e ~((R)-4 e)$

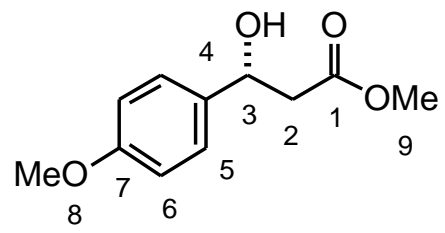

Following General Procedure 1, $243 \mu \mathrm{L}(2.0 \mathrm{mmol}, 1.0$ equiv $)$ of anisaldehyde was combined with $84 \mathrm{mg}(0.1 \mathrm{mmol}, 0.05$ equiv) of bisphosphoramide $(R, R)-3,252 \mu \mathrm{L}(2.2 \mathrm{mmol}$, 1.1 equiv) of $\mathrm{SiCl}_{4}$, and $523 \mu \mathrm{L}(2.4 \mathrm{mmol}, 1.2$ equiv) of 1-(tert-butyldimethylsilyloxy)-1methoxyethene (1) to yield, after purification by column chromatography (hexanes/EtOAc, 2/1), $410 \mathrm{mg}(97 \%)$ of $(R)-4 \mathrm{e}$ as a white solid. After recrystallization from benzene/hexanes, analytically pure, colorless needles were isolated.

Data for $(R)-4 \mathrm{e}$ :

mp: $\quad 67-68{ }^{\circ} \mathrm{C}$ (benzene/hexanes)

${ }^{1} \mathrm{H}$ NMR: $\quad\left(400 \mathrm{MHz}, \mathrm{CDCl}_{3}\right)$

7.30 (m, $2 \mathrm{H}, \mathrm{HC}(5)), 6.89$ (m, $2 \mathrm{H}, \mathrm{HC}(6)), 5.09$ (dt, $J=9.0,3.7,1 \mathrm{H}, \mathrm{HC}(3))$, 3.80 (s, $3 \mathrm{H}, \mathrm{HC}(8)), 3.72$ (s, $3 \mathrm{H}, \mathrm{HC}(9)), 3.10$ (d, $J=3.4,1 \mathrm{H}, \mathrm{OH}$ ), 2.73 (dd, $J$ $=16.4,8.7,1 \mathrm{H}, \mathrm{H} H \mathrm{C}(2)), 2.72(\mathrm{dd}, J=16.4,6.6,1 \mathrm{H}, H \mathrm{HC}(2))$ 
${ }^{13} \mathrm{C} \mathrm{NMR}: \quad\left(100 \mathrm{MHz}, \mathrm{CDCl}_{3}\right)$

172.74 (C(1)), $159.11(\mathrm{C}(7)), 134.50$ (C(4)), 126.84 (C(5)), 113.81 (C(6)), 69.84

(C(3)), $55.18(\mathrm{C}(8)), 51.79(\mathrm{C}(9)), 43.00(\mathrm{C}(2))$

IR: (neat)

3439 (s), 3001 (w), 2953 (m), 2837 (m), 1735 (s), 1612 (m), 1586 (w), 1514 (s), 1438 (m), 1358 (w), 1302 (m), 1248 (s), 1201 (m), 1174 (m), 1111 (w), 1032 (s), $833(\mathrm{~m})$.

MS: $\quad(\mathrm{EI}, 70 \mathrm{eV})$

$210\left(\mathrm{M}^{+}, 10\right), 161(10), 138(10), 137$ (100)

Opt. Rot.: $\quad[\alpha]_{\mathrm{D}}^{24}+14.4(\mathrm{c}=2.05, \mathrm{EtOH})$

TLC: $\quad R_{f} 0.20$ (hexanes/EtOAc, 2/1) [UV(254)/PMA]

SFC: $\quad t_{R}(R)-4 \mathbf{e}, 3.95(98.7 \%) ; t_{R}(S)-4 e, 4.45 \min (1.3 \%)$ (Chiralpak AD, 150 bar, $40{ }^{\circ} \mathrm{C}$, $6.0 \% \mathrm{CH}_{3} \mathrm{OH}$ in $\mathrm{CO}_{2}, 3.0 \mathrm{~mL} / \mathrm{min}, 220 \mathrm{~nm}$ )

Analysis: $\quad \mathrm{C}_{11} \mathrm{H}_{14} \mathrm{O}_{4}(210.23)$
Calcd:
C, 62.85;
$\mathrm{H}, 6.71 \%$
Found:
C, 62.83;
$\mathrm{H}, 6.75 \%$

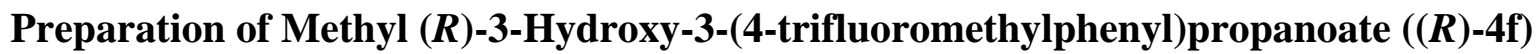

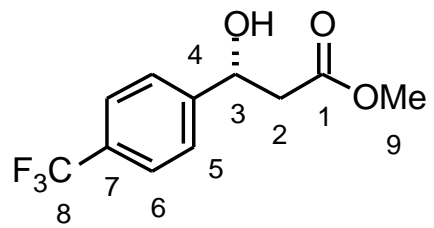

Following General Procedure 1, $273 \mu \mathrm{L} \quad(2.0 \mathrm{mmol}, \quad 1.0$ equiv $)$ of 4trifluoromethylbenzaldehyde was combined with $84 \mathrm{mg}(0.1 \mathrm{mmol}, 0.05$ equiv $)$ of bisphosphoramide $(R, R)-3,252 \mu \mathrm{L}\left(2.2 \mathrm{mmol}, 1.1\right.$ equiv) of $\mathrm{SiCl}_{4}$, and $523 \mu \mathrm{L}(2.4 \mathrm{mmol}, 1.2$ equiv) of 1-(tert-butyldimethylsilyloxy)-1-methoxyethene (1) to yield, after purification by column chromatography (hexanes/EtOAc, 2/1), $484 \mathrm{mg}(97 \%)$ of $(R)-\mathbf{4 f}$ as a white solid. Recrystallization from hexane afforded analytically pure, colorless needles.

Data for $(R)-\mathbf{4 f}:$

mp: $\quad 42-43{ }^{\circ} \mathrm{C}$ (hexane) 
ㅁN NMR: $\quad\left(400 \mathrm{MHz}, \mathrm{CDCl}_{3}\right)$

$7.58(\mathrm{~d}, J=8.3,2 \mathrm{H}, \mathrm{HC}(5)), 7.46$ (d, $J=8.0,2 \mathrm{H}, \mathrm{HC}(6)), 5.15$ (m, $1 \mathrm{H}, \mathrm{HC}(3))$,

$3.75(\mathrm{~d}, J=3.7,1 \mathrm{H}, \mathrm{OH}), 3.68$ (s, $3 \mathrm{H}, \mathrm{HC}(9)), 2.71(\mathrm{~m}, 2 \mathrm{H}, \mathrm{HC}(2))$

${ }^{13} \mathrm{C} \mathrm{NMR}: \quad\left(100 \mathrm{MHz}, \mathrm{CDCl}_{3}\right)$

172.72 (C(1)), 146.68 (C(4)), 130.10 (q, $J=32.0, \mathrm{C}(7)), 126.13$ (C(5)), 125.60

(C(6)), 124.22 (q, $J=271.8, \mathrm{C}(8)), 69.78$ (C(3)), 52.14 (C(9)), $43.15(\mathrm{C}(2))$

${ }^{19}$ F NMR: $\quad\left(376 \mathrm{MHz}, \mathrm{CDCl}_{3}\right)$

$-63.1\left(\mathrm{~F}_{3} \mathrm{C}(8)\right)$

IR: (neat)

3456 (s), 3005 (w), 2957 (m), 2851 (w), 1734 (s), 1621 (m), 1439 (m), 1416 (m), 1327 (s), 1272 (w), 1164 (s), 1124 (s), 1068 (s), 1017 (m), 845 (m)

MS: $\quad(\mathrm{CI})$

$248\left(\mathrm{M}^{+}, 8\right), 230$ (43), 223 (21), 212 (10), 184 (11), 175 (27), 168 (26), 157 (100)

Opt. Rot.: $\quad[\alpha]_{\mathrm{D}}^{24}+13.60(\mathrm{c}=2.27, \mathrm{EtOH})$

TLC: $\quad R_{f} 0.25$ (hexanes/EtOAc, 2/1) [UV(254)/PMA]

SFC: $\quad t_{R}(R)-\mathbf{4 f}, 3.75(95.7 \%) ; t_{R}(S)-\mathbf{4 f}, 4.14 \min (4.3 \%)$ (Chiralpak AS, 150 bar, $40{ }^{\circ} \mathrm{C}$, $7.5 \% \mathrm{CH}_{3} \mathrm{OH}$ in $\mathrm{CO}_{2}, 2.5 \mathrm{~mL} / \mathrm{min}, 220 \mathrm{~nm}$ )

Analysis: $\quad \mathrm{C}_{11} \mathrm{H}_{11} \mathrm{~F}_{3} \mathrm{O}_{3}(248.20)$
Calcd:
C, 53.23;
$\mathrm{H}, 4.47 \%$
Found:
C, 53.49;
$\mathrm{H}, 4.61 \%$

\section{Preparation of Methyl $(R)-3-H y d r o x y-5-p h e n y l-4-p e n t e n o a t e ~((R)-4 g)$}

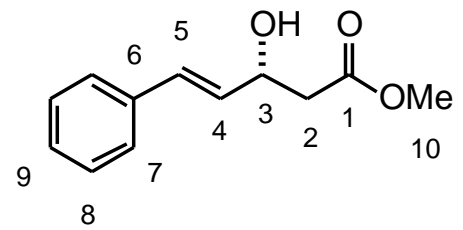

Following General Procedure 1, $252 \mu \mathrm{L}(2.0 \mathrm{mmol}, 1.0$ equiv) of cinnamaldehyde was combined with $84 \mathrm{mg}(0.1 \mathrm{mmol}, 0.05$ equiv) of bisphosphoramide $(R, R)-3,252 \mu \mathrm{L}(2.2 \mathrm{mmol}$, 1.1 equiv) of $\mathrm{SiCl}_{4}$, and $523 \mu \mathrm{L}(2.4 \mathrm{mmol}, 1.2$ equiv) of 1-(tert-butyldimethylsilyloxy)-1- 
methoxyethene (1) to yield, after purification by column chromatography (hexanes/EtOAc, 2/1), $393 \mathrm{mg}(95 \%)$ of $(R)-\mathbf{4 g}$ as a colorless oil. ${ }^{5}$

Data for $(R)-\mathbf{4 g}$ :

${ }^{1} \mathrm{H}$ NMR: $\quad\left(400 \mathrm{MHz}, \mathrm{CDCl}_{3}\right)$

$7.39(\mathrm{bd}, J=7.1,2 \mathrm{H}, 2 \times \mathrm{HC}(7)), 7.34(\mathrm{bt}, J=7.12 \mathrm{H}, 2 \times \mathrm{HC}(8)), 7.28$ (bt, $J=$ 6.1, $1 \mathrm{H}, \mathrm{HC}(9)), 6.67$ (d, $J=15.9,1 \mathrm{H}, \mathrm{HC}(5)), 6.24$ (dd, $J=15.9,6.1,1 \mathrm{H}$, HC(4)), 4.75 (m, $1 \mathrm{H}, \mathrm{HC}(3)), 3.74$ (s, 3 H, HC(10)), 3.23 (d, J = 4.6, $1 \mathrm{H}, \mathrm{HO}$ ), $2.67(\mathrm{dd}, J=16.4,1.2,1 \mathrm{H}, \mathrm{H} H \mathrm{C}(2)), 2.66$ (dd, $J=16.4,3.0,1 \mathrm{H}, H \mathrm{HC}(2))$

${ }^{13} \mathrm{C} \mathrm{NMR}: \quad(100 \mathrm{MHz}, \mathrm{CDCl} 3)$

$172.74(\mathrm{C}(1)), 136.50(\mathrm{C}(6)), 130.89(\mathrm{C}(5)), 130.06(\mathrm{C}(4)), 128.70(\mathrm{C}(7)), 127.95$ (C(9)), 126.67 (C(8)), 68.98 (C(3)), 52.03 (C(10)), $41.50(\mathrm{C}(2))$

IR: (neat)

3448 (s), 3058 (w), 3026 (m), 2952 (m), 1735 (s), 1494 (m), 1438 (s), 1403 (w), 1357 (m), 1283 (m), 1205 (m), 1166 (s), 1104 (m), 1030 (m), 969 (s), 751 (s), 694 (s)

Opt. Rot.: $\quad[\alpha]_{\mathrm{D}}^{24}-17.0(\mathrm{c}=2.07, \mathrm{EtOH})$

SFC: $\quad t_{R}(R)-\mathbf{4 g}, 3.61(96.9 \%) ; t_{R}(S)-\mathbf{4 g}, 4.24$ min (3.1\%) (Chiralpak OD, 150 bar, 40 ${ }^{\circ} \mathrm{C}, 7.5 \% \mathrm{CH}_{3} \mathrm{OH}$ in $\mathrm{CO}_{2}, 3.0 \mathrm{~mL} / \mathrm{min}, 220 \mathrm{~nm}$ )

\section{Preparation of Methyl $(R)-3-H y d r o x y-4-m e t h y l-5-p h e n y l-4-p e n t e n o a t e ~((R)-4 h)$}

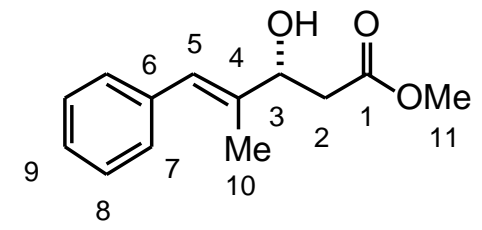

Following General Procedure 1, $279 \mu \mathrm{L} \quad(2.0 \mathrm{mmol}, \quad 1.0$ equiv $)$ of $\alpha$ methylcinnamaldehyde was combined with $84 \mathrm{mg}(0.1 \mathrm{mmol}, 0.05$ equiv) of bis-phosphoramide $(R, R)-3,252 \mu \mathrm{L}\left(2.2 \mathrm{mmol}, 1.1\right.$ equiv) of $\mathrm{SiCl}_{4}$, and $523 \mu \mathrm{L}(2.4 \mathrm{mmol}, 1.2$ equiv) of 1-(tertbutyldimethylsilyloxy)-1-methoxyethene (1) to yield, after purification by column 
chromatography (hexanes/EtOAc, 2/1), $432 \mathrm{mg}(98 \%)$ of $(R)-\mathbf{4 h}$ as a colorless oil. Kugelrohr distillation afforded an analytically pure, colorless oil. ${ }^{6}$

Data for $(R)-\mathbf{4 h}$ :

bp: $\quad 115^{\circ} \mathrm{C}\left(1.3 \times 10^{-4} \mathrm{mmHg}, \mathrm{ABT}\right)$

${ }^{1} \mathrm{H}$ NMR: $\quad\left(400 \mathrm{MHz}, \mathrm{CDCl}_{3}\right)$

7.35 (m, 2 H, HC(7)), 7.25 (m, 3 H, HC(8), HC(9)), 6.62 (bs, $1 \mathrm{H}, \mathrm{HC}(5)), 4.64$ (m, $1 \mathrm{H}, \mathrm{HC}(3)), 3.76$ (s, $3 \mathrm{H}, \mathrm{HC}(11)), 2.94$ (d, J = 3.4, 1 H, HO), 2.68 (m, 2 H, 2 x $\mathrm{HC}(2)), 1.90\left(\mathrm{~d}, J=1.5,3 \mathrm{H}, \mathrm{H}_{3} \mathrm{C}(10)\right)$

${ }^{13} \mathrm{C} \mathrm{NMR}: \quad\left(100 \mathrm{MHz}, \mathrm{CDCl}_{3}\right)$

$173.23(\mathrm{C}(1)), 138.26(\mathrm{C}(4)), 137.45(\mathrm{C}(6)), 129.20(\mathrm{C}(8)), 128.35(\mathrm{C}(9)), 126.83$ (C(7)), $126.26(\mathrm{C}(5)), 73.77(\mathrm{C}(3)), 52.18$ (C(11)), $40.28(\mathrm{C}(2)), 14.03(\mathrm{C}(10))$

IR: (neat) 3456 (s), 3024 (w), 2984 (w), 2952 (m), 2917 (w), 1736 (s), 1599 (m), 1492 (m), 1439 (s), 1347 (m), 1273 (s), 1169 (s), 1074 (m), 1042 (m), 990 (m), 920 (w), 870 (w), $753(\mathrm{~m})$

MS: $\quad(\mathrm{CI})$ $220\left(\mathrm{M}^{+}, 29\right), 202$ (17), 147 (100), 129 (74), 118 (63)

Opt. Rot.: $\quad[\alpha]_{\mathrm{D}}^{24}-13.70(\mathrm{c}=2.23, \mathrm{EtOH})$

TLC: $\quad R_{f} 0.31$ (hexanes/EtOAc, 2/1) [UV(254)/PMA]

SFC: $\quad t_{R}(R)-\mathbf{4 h}, 4.34(72.7 \%) ; t_{R}(S)-\mathbf{4 h}, 4.45 \min (27.3 \%)$ (Chiralpak AD, 150 bar, 40 ${ }^{\circ} \mathrm{C}, 6.0 \% \mathrm{CH}_{3} \mathrm{OH}$ in $\mathrm{CO}_{2}, 3.0 \mathrm{~mL} / \mathrm{min}, 220 \mathrm{~nm}$ )

Analysis: $\quad \mathrm{C}_{13} \mathrm{H}_{16} \mathrm{O}_{3}(220.26)$
Calcd:
C, 70.89;
$\mathrm{H}, 7.32 \%$
Found:
C, 70.61;
$\mathrm{H}, 7.33 \%$ 
Preparation of Methyl $(R)-3-H y d r o x y-3-(2-f u r y l) p r o p a n o a t e ~((R)-4 i)$

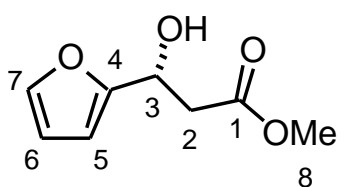

Following General Procedure 1, $165 \mu \mathrm{L}$ (2.0 mmol, 1.0 equiv) of furfural was combined with $84 \mathrm{mg}$ ( $0.1 \mathrm{mmol}, 0.05$ equiv) of bisphosphoramide $(R, R)-3,252 \mu \mathrm{L}$ (2.2 mmol, 1.1 equiv) of $\mathrm{SiCl}_{4}$, and $523 \mu \mathrm{L}$ (2.4 mmol, 1.2 equiv) of 1-(tert-butyldimethylsilyloxy)-1-methoxyethene (1) to yield, after purification by column chromatography (hexanes/EtOAc, 2/1), $320 \mathrm{mg}$ (94\%) of $(R)-\mathbf{4} \mathbf{i}$ as a colorless oil.

Data for $(R)-4 \mathbf{i}$ :

bp: $\quad 75^{\circ} \mathrm{C}\left(2.2 \times 10^{-4} \mathrm{mmHg}, \mathrm{ABT}\right)$

${ }^{1} \mathrm{H}$ NMR: $\quad\left(400 \mathrm{MHz}, \mathrm{CDCl}_{3}\right)$

7.38 (m, $1 \mathrm{H}, \mathrm{HC}(7)), 6.33$ (m, $1 \mathrm{H}, \mathrm{HC}(6)), 6.27$ (m, $1 \mathrm{H}, \mathrm{HC}(5)), 5.12$ (d, J = 4.4, 1 H, HC(3)), 3.73 (s, 3 H, HC(8)), 3.21 (bs, 1 H, HO), 2.90 (dd, J = 16.4, 6.3, $1 \mathrm{H}, \mathrm{H} H \mathrm{C}(2)), 2.88(\mathrm{dd}, J=16.4,5.4,1 \mathrm{H}, H \mathrm{HC}(2))$

${ }^{13} \mathrm{C} \mathrm{NMR}: \quad\left(100 \mathrm{MHz}, \mathrm{CDCl}_{3}\right)$

$172.64(\mathrm{C}(1)), 154.83$ (C(4), $142.56(\mathrm{C}(7)), 110.52$ (C(6)), $106.62(\mathrm{C}(5)), 64.38$ (C(3)), $52.28(\mathrm{C}(8)), 39.78(\mathrm{C}(2))$

IR: (neat)

3460 (s), 2955 (m), 1736 (s), 1504 (w), 1439 (m), 1363 (m), 1284 (m), 1211 (m), $1164(\mathrm{~m}), 1066(\mathrm{w}), 1030(\mathrm{~m}), 1011(\mathrm{~m}), 741(\mathrm{~m})$

MS: $\quad(E I, 70 \mathrm{eV})$

$170\left(\mathrm{M}^{+}, 18\right), 110(14), 97$ (100), 84 (46), 71 (13)

Opt. Rot.: $\quad[\alpha]_{\mathrm{D}}^{24}+22.65(\mathrm{c}=2.15, \mathrm{EtOH})$

TLC: $\quad R_{f} 0.30$ (hexanes/EtOAc, 2/1) [UV(254)/PMA]

SFC: $\quad t_{R}(R)-4 \mathbf{i}, 4.02(93.4 \%) ; t_{R}(S)-4 \mathbf{i}, 4.55 \min (6.6 \%)$ (Chiralpak AS, 150 bar, $40{ }^{\circ} \mathrm{C}$, $7.5 \% \mathrm{CH}_{3} \mathrm{OH}$ in $\mathrm{CO}_{2}, 2.5 \mathrm{~mL} / \mathrm{min}, 220 \mathrm{~nm}$ ) 
Preparation of Methyl $(R)-3-H y d r o x y-3-c y c l o h e x y l p r o p a n o a t e ~((R)-4 j)$

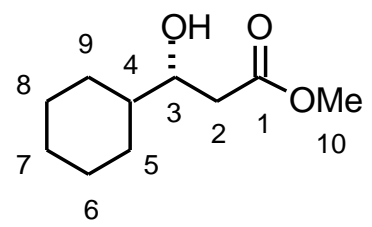

A flame-dried, 20-mL, 2-neck flask containing a solution of $168 \mathrm{mg}(0.2 \mathrm{mmol}, 0.1$ equiv) of bisphosphoramide $(R, R)-3$ in $\mathrm{CH}_{2} \mathrm{Cl}_{2}(10 \mathrm{~mL})$ was cooled to $-78{ }^{\circ} \mathrm{C}$ under nitrogen. Into this reaction mixture was added $242 \mu \mathrm{L} \quad(2.0 \mathrm{mmol}, 1.0$ equiv $)$ of cyclohexanecarboxaldehyde. To the resulting mixture $252 \mu \mathrm{L}\left(2.2 \mathrm{mmol}, 1.1\right.$ equiv) of $\mathrm{SiCl}_{4}$ was added and the reaction mixture was allowed to stir at $-78{ }^{\circ} \mathrm{C}$ for $5 \mathrm{~min}$. Then $523 \mu \mathrm{L}(2.4$ mmol, 1.2 equiv) of 1-(tert-butyldimethylsilyloxy)-1-methoxyethene (1) was added dropwise (over $5 \mathrm{~min}$ ) the resulting mixture was allowed to stir at $-78^{\circ} \mathrm{C}$ (bath temperature) for $6 \mathrm{~h}$. The cold reaction mixture was then poured into a rapidly stirring $1 / 1$ sat. aq. $\mathrm{KF} / 1.0 \mathrm{M} \mathrm{KH}_{2} \mathrm{PO}_{4}$ solution $(50 \mathrm{~mL})$. This biphasic mixture was stirred vigorously for $2 \mathrm{~h}$ after which the aqueous layer was washed with $\mathrm{CH}_{2} \mathrm{Cl}_{2}(3 \times 50 \mathrm{~mL})$. The combined organic extracts were dried over $\mathrm{Na}_{2} \mathrm{SO}_{4}$, filtered and the filtrate was concentrated in vacuo. The residue was purified by column chromatography (hexanes/EtOAc, 2/1). The product-containing fractions were combined and the solvent was removed in vacuo to yield $321 \mathrm{mg}(86 \%)$ of $(R)-\mathbf{4 j}$ as a colorless oil. ${ }^{6}$ Analysis of the enantiomeric composition was preformed on the trifluoroacetate derivative.

Data for $(R)-\mathbf{4 j}$ :

${ }^{1} \mathrm{H}$ NMR: $\quad\left(400 \mathrm{MHz}, \mathrm{CDCl}_{3}\right)$

3.78 (m, $1 \mathrm{H}, \mathrm{HC}(3)), 3.71$ (s, $3 \mathrm{H}, \mathrm{HC}(10)), 2.81$ (d, $J=4.1,1 \mathrm{H}, \mathrm{HO}), 2.52$ (dd, $J=16.1,2.7,1 \mathrm{H}, \mathrm{HHC}(2)), 2.43(\mathrm{dd}, J=16.1,9.4,1 \mathrm{H}, H \mathrm{HC}(2)), 1.86(\mathrm{bd}, 1 \mathrm{H}$, C(9)), 1.80-1.72 (m, 2 H, 2 x HC(8)), 1.66 (m, 2 H, 2 x HC(5)) 1.42-1.32 (m, 1 H, $\mathrm{HC}(4)), 1.30$ - 0.95 (m, 5 H, 2 x HC(7), HC(9), 2 x HC(6))

${ }^{13} \mathrm{C} \mathrm{NMR}: \quad\left(100 \mathrm{MHz}, \mathrm{CDCl}_{3}\right)$

$174.20(\mathrm{C}(1)), 72.35(\mathrm{C}(3)), 52.10(\mathrm{C}(10)), 43.23$ (C(4)), $38.56(\mathrm{C}(2)), 28.98$ (C(7)), 28.43 (C(5)), 26.60 (C(6)), $26.34(\mathrm{C}(8)), 26.23$ (C(9)) 
IR: $\quad$ (neat)

3462 (s), 2926 (s), 2853 (s), 1737 (s), 1439 (s), 1407 (w), 1366 (w), 1311 (w), $1281(\mathrm{w}), 1192(\mathrm{w}), 1168(\mathrm{~m}), 992$ (m), $892(\mathrm{~m}), 843(\mathrm{w})$

Opt. Rot.: $\quad[\alpha]_{\mathrm{D}}^{24}+17.5(\mathrm{c}=2.84, \mathrm{EtOH})$

GC: $\quad t_{R}(S)-8,7.73(5.8 \%) ; t_{R}(R)-8,8.20 \min (94.2 \%)\left(\mathrm{GTA}, 16 \mathrm{psi}, 100{ }^{\circ} \mathrm{C}\right.$ isothermal)

\section{Preparation of Methyl $(R)-3-H y d r o x y-5-p h e n y l p e n t a n o a t e ~((S)-4 k)$}

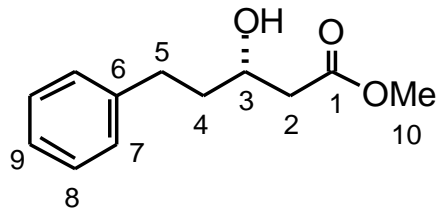

A flame-dried, 20-mL, 2-neck flask containing a solution of $168 \mathrm{mg}(0.2 \mathrm{mmol}, 0.1$ equiv) of bisphosphoramide $(R, R)-3$ in $\mathrm{CH}_{2} \mathrm{Cl}_{2}(10 \mathrm{~mL})$ was cooled to $-78{ }^{\circ} \mathrm{C}$ under nitrogen. To this reaction mixture was added $263 \mu \mathrm{L}$ ( $2.0 \mathrm{mmol}, 1.0$ equiv) of hydrocinnamaldehyde. To the resulting mixture was added $252 \mu \mathrm{L}\left(2.2 \mathrm{mmol}, 1.1\right.$ equiv) of $\mathrm{SiCl}_{4}$ and the reaction mixture was allowed to stir at $-78{ }^{\circ} \mathrm{C}$ for $5 \mathrm{~min}$. Then $523 \mu \mathrm{L}(2.4 \mathrm{mmol}, 1.2$ equiv) of 1 -(tertbutyldimethylsilyloxy)-1-methoxyethene (1) was added dropwise (over $5 \mathrm{~min}$ ) to the reaction mixture. The resulting mixture was allowed to stir at $-78{ }^{\circ} \mathrm{C}$ (bath temperature) for $6 \mathrm{~h}$. The cold reaction mixture was then poured into a rapidly stirring $1 / 1$ sat. aq. $\mathrm{KF} / 1.0 \mathrm{M} \mathrm{KH}_{2} \mathrm{PO}_{4}$ solution $(50 \mathrm{~mL})$. This biphasic mixture was stirred vigorously for $2 \mathrm{~h}$ after which the aqueous layer was washed with $\mathrm{CH}_{2} \mathrm{Cl}_{2}(3 \times 50 \mathrm{~mL})$. The combined organic extracts were dried over $\mathrm{Na}_{2} \mathrm{SO}_{4}$, filtered and the filtrate was concentrated in vacuo. The residue was purified by column chromatography (hexanes/EtOAc, 2/1). The product-containing fractions were combined and the solvent was removed in vacuo to yield $300 \mathrm{mg}(72 \%)$ of (S)-4k as a colorless oil. ${ }^{7}$ 


\section{Data for $(S)-\mathbf{4 k}$ :}

${ }^{1} \mathrm{H}$ NMR: $\quad\left(400 \mathrm{MHz}, \mathrm{CDCl}_{3}\right)$

7.28-7.18 (m, 5 H, HC(7,8,9)), 4.01 (m, 1 H, HC(3)), 3.70 (s, 3 H, H (d, $J=3.6,1 \mathrm{H}, \mathrm{OH}), 2.82$ (m, $1 \mathrm{H}, \mathrm{H} H \mathrm{C}(5)), 2.69$ (m, $1 \mathrm{H}, \mathrm{HHC}(5)), 2.50$ (dd, $J$ $=16.5,3.4,1 \mathrm{H}, \mathrm{H} H \mathrm{C}(2)), 2.46(\mathrm{dd}, J=16.5,8.7,1 \mathrm{H}, \mathrm{H} H \mathrm{C}(2)), 1.84(\mathrm{~m}, 1 \mathrm{H}$, $\mathrm{H} H \mathrm{C}(4)), 1.74(\mathrm{~m}, 1 \mathrm{H}, \mathrm{H} H \mathrm{C}(4))$

${ }^{13} \mathrm{C} \mathrm{NMR}: \quad\left(100 \mathrm{MHz}, \mathrm{CDCl}_{3}\right)$

$173.31(\mathrm{C}(1)), 141.61(\mathrm{C}(6)), 128.38$ (C(7)), 128.35 (C(8)), 125.83 (C(9)), 67.12 (C(3)), 51.72 (C(10)), $41.07(\mathrm{C}(5)), 38.05(\mathrm{C}(2)), 31.69(\mathrm{C}(4))$

IR: (neat)

3450 (w), 2951 (s), 2929 (s), 2862 (w), 1737 (s), 1603 (w), 1496 (m), 1454 (s), 1439 (s), 1409 (m), 1359 (m), 1303 (s), 1254 (s), 1198 (s), 1177 (s), 1158 (s), 1082 (s), 1060 (s), 1045 (s), 1031 (m), 1002 (m)

Opt. Rot.: $\quad[\alpha]_{\mathrm{D}}^{24}+17.5(\mathrm{c}=2.84, \mathrm{EtOH})$

SFC: $\quad t_{R}(S)-\mathbf{4 k}, 3.46(90.7 \%) ; t_{R}(R)-4 \mathbf{k}, 4.13$ min (9.3\%) (Chiralpak OD, 150 bar, 40 ${ }^{\circ} \mathrm{C}, 5.0 \% \mathrm{CH}_{3} \mathrm{OH}$ in $\mathrm{CO}_{2}, 3.0 \mathrm{~mL} / \mathrm{min}, 220 \mathrm{~nm}$ )

\section{(E)-[(1-Methoxy)-propenyloxy)-tert-butyldimethylsilane $((E)-5 a)$}

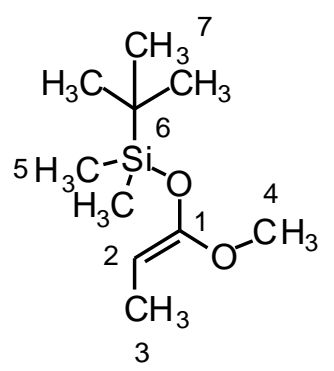

To a flame-dried, 100-mL, 3-neck round-bottom flask was added $1.54 \mathrm{~mL}$ of diisopropylamine ( $11 \mathrm{mmol}, 1.1$ equiv) and $14 \mathrm{~mL}$ of THF. The solution was cooled to $0{ }^{\circ} \mathrm{C}$ in a ice-water bath and then $9.2 \mathrm{~mL}$ of $n$-BuLi (1.2 $\mathrm{M}$ in hexane, $11 \mathrm{mmol}, 1.1$ equiv) was added slowly over five min. The resulting solution was stirred for $30 \mathrm{~min}$ at $0{ }^{\circ} \mathrm{C}$ prior to cooling to $-78{ }^{\circ} \mathrm{C}$ in a dry ice/isopropanol bath. Then $0.96 \mathrm{~mL}$ of methyl propanoate (10 mmol, 1.0 equiv) was added slowly over five min. The resulting solution was stirred for an additional $30 \mathrm{~min}$ at 
$-78{ }^{\circ} \mathrm{C}$. Then a solution of $1.65 \mathrm{~g}$ of $\mathrm{TBSCl}(11 \mathrm{mmol}, 1.1$ equiv) and $4 \mathrm{~mL}$ of HMPA (22 mmol, 2.2 equiv) in $4 \mathrm{~mL}$ of THF was added slowly via cannula. The dry ice/isopropanol bath was then removed and the solution allowed to stir for $1 \mathrm{~h}$ at room temperature. The yellow solution was diluted with cold pentane $(50 \mathrm{~mL})$ and was washed with cold water $(3 \mathrm{x} 75 \mathrm{~mL})$. The organic layer was dried over $\mathrm{Na}_{2} \mathrm{SO}_{4}(10 \mathrm{~g})$, and filtered through Celite and the filtrate was concentrated in vacuo. The residue was purified by Kugelrohr distillation to yield $0.852 \mathrm{~g}$ (42\%, $82 / 18 E / Z)$ of $\mathbf{5 a}$ as a colorless oil. ${ }^{8}$

\section{Data for $(E)-5 \mathbf{a}$ :}

bp: $\quad 100{ }^{\circ} \mathrm{C}(1 \mathrm{mmHg}, \mathrm{ABT})$

${ }^{1} \mathrm{H}$ NMR: $\quad\left(500 \mathrm{MHz}, \mathrm{CDCl}_{3}\right)$

3.65 (q, $J=6.59,1 \mathrm{H}, \mathrm{HC}(2)), 3.54\left(\mathrm{~s}, 3 \mathrm{H}, \mathrm{H}_{3} \mathrm{C}(4)\right), 1.49(\mathrm{~d}, J=6.59,3 \mathrm{H}$, $\left.\mathrm{H}_{3} \mathrm{C}(3)\right), 0.941$ (s, $\left.9 \mathrm{H}, \mathrm{H}_{3} \mathrm{C}(7)\right), 0.175$ (s, $\left.6 \mathrm{H}, \mathrm{H}_{3} \mathrm{C}(5)\right)$

${ }^{13} \mathrm{C} \mathrm{NMR}: \quad\left(100 \mathrm{MHz}, \mathrm{CDCl}_{3}\right)$

154.32 (C(1)), 78.83 (C(2)), 55.06 (C(4)), 25.86 (C(3)), 18.37 (C(7)), 9.67 (C(6)), $-4.77(\mathrm{C}(5))$

IR: (neat)

2597 (s), 2932 (s), 2860 (s), 1686 (s), 1472 (m), 1254 (s), 1221 (s), 1177 (m), $1110(\mathrm{~m})$

$\underline{\mathrm{MS}}: \quad(\mathrm{EI}, 70 \mathrm{eV})$

$203\left(\mathrm{M}^{+}, 31\right), 189$ (43), 163 (12), 147 (43), 131 (28), 115 (15), 89 (38), 73 (100)

\section{(E)-[(1-Phenoxy)propenyloxy]-tert-butyldimethylsilane $((E)-5 c)$}

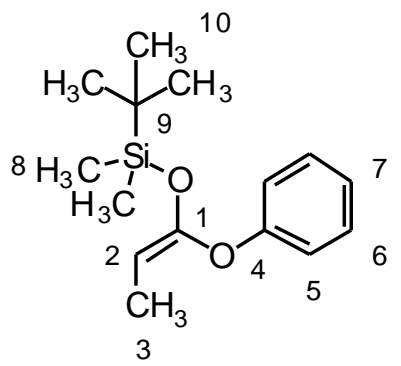

To a flame-dried, 250-mL, 3-neck round-bottom flask was added $3.08 \mathrm{~mL}$ of diisopropylamine (22 mmol, 1.1 equiv) and $28 \mathrm{~mL}$ of THF. The solution was cooled to $0{ }^{\circ} \mathrm{C}$ under nitrogen in an ice-water bath and then $14 \mathrm{~mL}$ of $n$-BuLi (1.55 $\mathrm{M}$ in hexane, $22 \mathrm{mmol}, 1.1$ 
equiv) was added slowly over $5 \mathrm{~min}$. The resulting solution was stirred for $30 \mathrm{~min}$ at $0{ }^{\circ} \mathrm{C}$ prior to cooling to $-78{ }^{\circ} \mathrm{C}$ in a dry ice/isopropanol bath. Then $2.86 \mathrm{~mL}$ of propanoic acid phenyl ester (20 mmol, 1.0 equiv) was added slowly over $5 \mathrm{~min}$. The resulting solution was stirred for an additional $30 \mathrm{~min}$ at $-78{ }^{\circ} \mathrm{C}$. Then a solution of $3.3 \mathrm{~g}$ of TBSCl (22 mmol, 1.1 equiv) and $8 \mathrm{~mL}$ of HMPA (44 mmol, 2.2 equiv) in $8 \mathrm{~mL}$ of THF was added slowly via cannula. The dry ice/isopropanol bath was then removed and the solution was allowed to stir for $1 \mathrm{~h}$ at room temperature. The yellow solution was diluted with cold pentane $(100 \mathrm{~mL})$ and washed with cold water $(3 \times 75 \mathrm{~mL})$. The organic layer was dried over $\mathrm{Na}_{2} \mathrm{SO}_{4}(25 \mathrm{~g})$, filtered through Celite and the filtrate was concentrated in vасио. The residue was purified by Kugelrohr distillation to yield $2.64 \mathrm{~g}(50 \%, 94 / 6 \mathrm{E} / \mathrm{Z})$ of $\mathbf{5 c}$ as a colorless oil.

\section{Data for $(E)-5 \mathbf{c}:$}

bp: $\quad 125^{\circ} \mathrm{C}(1 \mathrm{mmHg}, \mathrm{ABT})$

${ }^{1} \mathrm{H}$ NMR: $\quad\left(400 \mathrm{MHz}, \mathrm{CDCl}_{3}\right)$

7.26 (m, 2 H, HC(5)), 7.0 (m, 3 H, HC(6,7)), 4.26 (q, J = 6.56, 1 H, HC(2)), 1.52

$\left(\mathrm{d}, J=6.64,3 \mathrm{H}, \mathrm{H}_{3} \mathrm{C}(3)\right), 0.819$ (s, $\left.9 \mathrm{H}, \mathrm{H}_{3} \mathrm{C}(10)\right), 0.09$ (s, $6 \mathrm{H}, \mathrm{H}_{3} \mathrm{C}(8)$ )

${ }^{13} \mathrm{C} \mathrm{NMR}: \quad\left(100 \mathrm{MHz}, \mathrm{CDCl}_{3}\right)$

155.57 (C(4)), 150.18 (C(1)), $129.5(\mathrm{C}(6)), 122.03$ (C(7)), 116.13 (C(5)), 86.57

(C(2)), $25.36(\mathrm{C}(3)), 17.91(\mathrm{C}(10)), 9.72(\mathrm{C}(9)),-5.04(\mathrm{C}(8))$

IR: (neat)

2957 (s), 2931 (s), 2887 (m), 2860 (s), 1695 (s), 1596 (s), 1491 (s), 1472 (s), 1297

(m)

MS: $\quad(\mathrm{EI}, 70 \mathrm{eV})$

264 (M+17), 207 (24), 193 (28), 171 (29), 151 (26), 137 (18), 115 (21), 105 (10),

73 (100) 


\section{(E)-[(1-tert-Butoxy)propenyloxy]-tert-butyldimethylsilane $((E)-5 d)$}

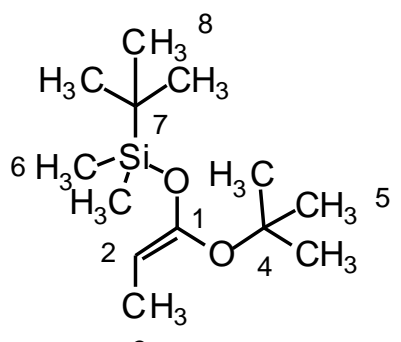

3

To a flame-dried, 250-mL, 3-neck round-bottom flask was added $6.2 \mathrm{~mL}$ of diisopropylamine ( $44 \mathrm{mmol}, 1.1$ equiv) and $56 \mathrm{~mL}$ of THF. The solution was cooled to $0{ }^{\circ} \mathrm{C}$ under nitrogen in an ice-water bath and then $28 \mathrm{~mL}$ of $n$-BuLi (1.58 $\mathrm{M}$ in hexane, $44 \mathrm{mmol}, 1.1$ equiv) was added slowly over $5 \mathrm{~min}$. The resulting solution was stirred for $30 \mathrm{~min}$ at $0{ }^{\circ} \mathrm{C}$ prior to cooling to $-78{ }^{\circ} \mathrm{C}$ in a dry ice/isopropanol bath. Then $6.0 \mathrm{~mL}$ of tert-butyl propanoate (40 mmol, 1.0 equiv) was added slowly over $5 \mathrm{~min}$. The resulting solution was stirred for an additional $30 \mathrm{~min}$ at $-78{ }^{\circ} \mathrm{C}$. Then a solution of $6.6 \mathrm{~g}$ of $\mathrm{TBSCl}$ (44 mmol, 1.1 equiv) and $16 \mathrm{~mL}$ of HMPA ( $88 \mathrm{mmol}, 2.2$ equiv) in $8 \mathrm{~mL}$ of THF was added slowly via cannula. The dry ice/isopropanol bath was then removed and the solution allowed to stir for $1 \mathrm{~h}$ at room temperature. The yellow solution was diluted with cold pentane $(100 \mathrm{~mL})$ and washed with cold water $(3 \times 75 \mathrm{~mL})$. The organic layer was dried over $\mathrm{Na}_{2} \mathrm{SO}_{4}(50 \mathrm{~g})$, filtered through Celite and the filtrate was concentrated in vacuo. The residue was purified by short path distillation to yield $6.8 \mathrm{~g}(70 \%, 95 / 5 \mathrm{E} / \mathrm{Z})$ of $\mathbf{5} \mathbf{d}$ as a colorless oil.

\section{Data for $(E)-5 \mathbf{d}$ :}

bp: $\quad 70^{\circ} \mathrm{C}(1 \mathrm{mmHg}, \mathrm{ABT})$

${ }^{1} \mathrm{H}$ NMR: $\quad\left(399 \mathrm{MHz}, \mathrm{CDCl}_{3}\right)$

$3.93(\mathrm{q}, J=6.59,1 \mathrm{H}, \mathrm{HC}(2)), 1.48\left(\mathrm{~d}, J=6.59,3 \mathrm{H}, \mathrm{H}_{3} \mathrm{C}(3)\right), 1.32(\mathrm{~s}, 9 \mathrm{H}$, $\left.\mathrm{H}_{3} \mathrm{C}(5)\right), 0.92\left(\mathrm{~s}, 9 \mathrm{H}, \mathrm{H}_{3} \mathrm{C}(8)\right), 0.15$ (s, $\left.6 \mathrm{H}, \mathrm{H}_{3} \mathrm{C}(6)\right)$

${ }^{13} \mathrm{C} \mathrm{NMR}: \quad\left(100 \mathrm{MHz}, \mathrm{CDCl}_{3}\right)$

$152.21(\mathrm{C}(1)), 86.04(\mathrm{C}(2), 78.32$ (C(4)), 29.09 (C(3)), $25.74(\mathrm{C}(5)), 17.99(\mathrm{C}(8))$, $10.89(\mathrm{C}(7)),-4.81(\mathrm{C}(6))$

IR: (neat)

2997 (s), 2960 (s), 2932 (s), 2861 (s), 1678 (s), 1256 (s), 1207 (s), 1152 (s), 1110 (m), $861(\mathrm{~s}), 839$ (s), $781(\mathrm{~m})$ 
MS: $\quad(E I, 70 \mathrm{eV})$

245 (15), 244 (10), 190 (13), 189 (76), 188 (60), 187 (20), 173 (30), 132 (15), 131

(100), 130 (11), 115 (11), 75 (90), 73 (45), 57 (56)

Analysis: $\quad \mathrm{C}_{13} \mathrm{H}_{27} \mathrm{O}_{2} \mathrm{Si}(244.19)$
Calcd:
C, 63.87;
$\mathrm{H}, 11.55 \%$
Found:
C, 63.91;
$\mathrm{H}, 11.58 \%$

\section{(Z)-[(1-tert-Butoxy)-propenyloxy]-tert-butyldimethylsilane ((Z)-5d)}

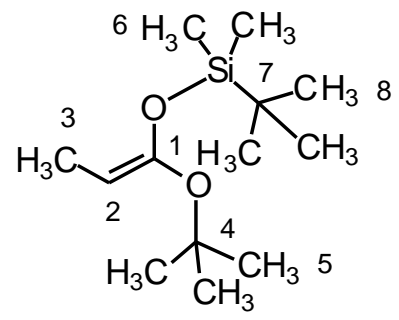

To a flame-dried, 250-mL, 3-neck round-bottom flask was added $2.46 \mathrm{~mL}$ of hexamethyldisilazane ( $11 \mathrm{mmol}, 1.0$ equiv) and $18 \mathrm{~mL}$ of THF. The solution was cooled to $0{ }^{\circ} \mathrm{C}$ under nitrogen in an ice-water bath and then $7.15 \mathrm{~mL}$ of $n$-BuLi $(1.55 \mathrm{M}$ in hexane, $11 \mathrm{mmol}, 1.1$ equiv) was added slowly over $5 \mathrm{~min}$. The resulting solution was stirred for $30 \mathrm{~min}$ at $0{ }^{\circ} \mathrm{C}$ prior to cooling to $-78{ }^{\circ} \mathrm{C}$ in a dry ice/isopropanol bath. To this solution was added $12 \mathrm{~mL}$ of HMPA (88 mmol, 2.2 equiv). Then $1.65 \mathrm{~mL}$ of tert-butyl propanoate (11 mmol, 1.0 equiv) was added slowly over $5 \mathrm{~min}$. The resulting solution was stirred for an additional $1 \mathrm{~h}$ at $-78{ }^{\circ} \mathrm{C}$. Then a solution of $1.8 \mathrm{~g}$ of TBSCl (12 mmol, 1.1 equiv) in $8 \mathrm{~mL}$ of THF was added slowly via cannula. The dry ice/isopropanol bath was then removed and the solution allowed to stir for $1 \mathrm{~h}$ at room temperature. The yellow solution was diluted with cold pentane $(100 \mathrm{~mL})$ and washed with cold water $(3 \times 75 \mathrm{~mL})$. The organic layer was dried over $\mathrm{Na}_{2} \mathrm{SO}_{4}(10 \mathrm{~g})$, filtered through Celite and the filtrate was concentrated in vacuo. The residue was purified by short path distillation to yield $1.72 \mathrm{~g}(64 \%, 12 / 88 \mathrm{E} / \mathrm{Z})$ of $\mathbf{5 d}$ as a colorless oil.

Data for $(Z)-\mathbf{5 d}$ :

bp: $\quad 70^{\circ} \mathrm{C}(1 \mathrm{mmHg}, \mathrm{ABT})$ 
${ }^{1} \mathrm{H}$ NMR: $\quad\left(500 \mathrm{MHz}, \mathrm{CDCl}_{3}\right)$

$3.98(\mathrm{q}, J=6.0,1 \mathrm{H}, \mathrm{HC}(2)), 1.50(\mathrm{~d}, J=6.64,3 \mathrm{H}, \mathrm{HC}(3)), 1.28$ (s, $9 \mathrm{H}$, $\left.\mathrm{H}_{3} \mathrm{C}(5)\right), 0.948$ (s, $\left.9 \mathrm{H}, \mathrm{H}_{3} \mathrm{C}(8)\right), 0.158$ (s, $\left.6 \mathrm{H}, \mathrm{H}_{3} \mathrm{C}(6)\right)$

${ }^{13} \mathrm{C} \mathrm{NMR}: \quad\left(125 \mathrm{MHz}, \mathrm{CDCl}_{3}\right)$

$151.63(\mathrm{C}(1)), 87.02$ (C(2)), 78.22 (C(4)), 29.10 (C(3)), 28.48 (C(5)), 17.99 $(\mathrm{C}(8)), 10.70(\mathrm{C}(7)),-4.21(\mathrm{C}(6))$

IR: (neat)

2978 (s), 2959 (s), 2931 (s), 2860 (s), 1680 (s), 1473 (m), 1389 (m), 1366 (m), 1306 (s), 1254 (s), 1209 (m), 1146 (s), 1104 (m), 1049 (s)

$\underline{\mathrm{MS}}$ : $\quad(\mathrm{EI}, 70 \mathrm{eV})$

$244\left(1.00, \mathrm{M}^{+}\right), 188$ (28), 173 (7), 157 (4), 147 (7), 131 (56), 115 (16), 75 (100), $57(75)$

General Procedure 2. Addition of Propionate-derived Silyl Ketene Acetals to Aromatic Aldehydes.

Preparation of tert-Butyl (2S,3R)-3-Hydroxy-2-Methyl-3-Phenylpropanate ((2S,3R)-6da)

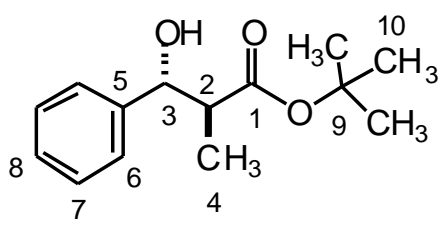

A flame-dried, 10-mL, 2-neck flask containing a solution of $8.4 \mathrm{mg}(0.01 \mathrm{mmol}, 0.01$ equiv) of bisphosphoramide $(R, R)-3$ in $\mathrm{CH}_{2} \mathrm{Cl}_{2}(5 \mathrm{~mL})$ was cooled to $-78{ }^{\circ} \mathrm{C}$ under nitrogen and then $102 \mu \mathrm{L}$ (1.0 mmol, 1.0 equiv) of benzaldehyde was added. To the resulting solution was added $123 \mu \mathrm{L}$ (1.1 mmol, 1.1 equiv) of $\mathrm{SiCl}_{4}$ and the reaction mixture was allowed to stir at -78 ${ }^{\circ} \mathrm{C}$ for $5 \mathrm{~min}$. Then $293 \mathrm{mg}(1.2 \mathrm{mmol}, 1.2$ equiv) of (E)-1-[(tert-butoxy)propenyl]-tertbutyldimethylsilane $((E)-\mathbf{5 d})$ was added dropwise (over $5 \mathrm{~min}$ ) to the reaction mixture. The resulting mixture was allowed to stir at $-78{ }^{\circ} \mathrm{C}$ (bath temperature) for $3 \mathrm{~h}$ whereupon the cold reaction mixture was poured into a rapidly stirring 1/1 sat. aq. KF/1.0 M KH $\mathrm{PO}_{4}$ solution (20 $\mathrm{mL}$ ). This biphasic mixture was stirred vigorously for $1 \mathrm{~h}$ before filtration through Celite. The aqueous layer was washed with $\mathrm{CH}_{2} \mathrm{Cl}_{2}(3 \times 50 \mathrm{~mL})$. The combined organic extracts were 
washed with brine $(50 \mathrm{~mL})$, dried over $\mathrm{Na}_{2} \mathrm{SO}_{4}(2 \mathrm{~g})$, filtered and the filtrate was concentrated in vасио. The residue was purified by Kugelrohr distillation to yield $217 \mathrm{mg}(93 \%)$ of $(2 S, 3 R)$-6da as a colorless oil.

\section{Data for $(2 S, 3 R)$-6da:}

bp: $\quad 150{ }^{\circ} \mathrm{C}(0.01 \mathrm{mmHg}, \mathrm{ABT})$

글 $\quad$ (499 $\mathrm{MHz}, \mathrm{CDCl}_{3}$ )

7.40-7.20 (m, $5 \mathrm{H}, \mathrm{HC}(6,7,8)), 4.70(\mathrm{dd}, J=7.9,4.9,1 \mathrm{H}, \mathrm{HC}(3)), 3.19$ (d, $J=$ 4.9, $1 \mathrm{H}, \mathrm{OH}$ ), 2.70 (pent, $J=7.29,1 \mathrm{H}, \mathrm{HC}(2)), 1.44$ (s, $\left.9 \mathrm{H}, \mathrm{H}_{3} \mathrm{C}(10)\right), 1.01$ (d, $J$ $\left.=7.29,3 \mathrm{H}, \mathrm{H}_{3} \mathrm{C}(4)\right)$

${ }^{13} \mathrm{C} \mathrm{NMR}: \quad\left(125 \mathrm{MHz}, \mathrm{CDCl}_{3}\right)$

$175.3(\mathrm{C}(1)), 141.8(\mathrm{C}(5)), 128.3(\mathrm{C}(7)), 127.8(\mathrm{C}(8)), 126.3(\mathrm{C}(6)), 81.2(\mathrm{C}(9))$, $76.3(\mathrm{C}(3)), 47.68(\mathrm{C}(2)), 27.9(\mathrm{C}(10)), 14.6(\mathrm{C}(4))$

IR: (neat)

3455 (w), 2978 (s), 2936 (m), 1727 (s), 1456 (m), 1368 (s), 1253 (m), 1154 (s), $1021(\mathrm{~m}), 701(\mathrm{~m})$

MS: $\quad(\mathrm{FAB})$

$237\left(\mathrm{M}^{+}, 6\right), 181$ (11), 163 (25), 155 (43), 135 (50), 119 (100)

Opt. Rot.: $\quad[\alpha]_{\mathrm{D}}^{24}+36.70(\mathrm{c}=1.0, \mathrm{EtOH})$

TLC: $\quad R_{f} 0.35$ (hexanes/EtOAc, 8/2) [UV(254)/ $\left.\mathrm{KMnO}_{4}\right]$

SFC: $\quad t_{R}(2 S, 3 R)-6 \mathbf{d a}, 2.32(>99.9 \%) ; t_{R}(2 R, 3 S)-\mathbf{6 d a}, 2.81 \mathrm{~min}(<0.1 \%)$ (Chiralpak AS, 150 bar, $40{ }^{\circ} \mathrm{C}, 1.0 \% \mathrm{CH}_{3} \mathrm{OH}$ in $\mathrm{CO}_{2}, 3.5 \mathrm{~mL} / \mathrm{min}, 220 \mathrm{~nm}$ )

Analysis: $\quad \mathrm{C}_{14} \mathrm{H}_{20} \mathrm{O}_{3}(230.26)$
Calcd:
C, 71.16;
$\mathrm{H}, 8.53 \%$
Found:
C, 70.93;
H, $8.46 \%$ 
Preparation of tert-Butyl (2S,3R)-3-Hydroxy-2-methyl-3-(1-naphthalenyl)propanoate $((2 S, 3 R)-6 \mathrm{db})$

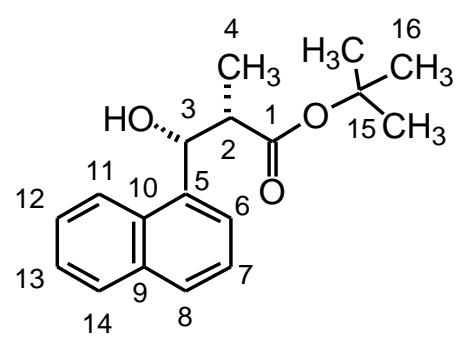

Following General Procedure 2, $136 \mu \mathrm{L}$ (1.0 mmol, 1.0 equiv.) of 1-naphthaldehyde was combined with $8.4 \mathrm{mg}(0.01 \mathrm{mmol}, 0.01$ equiv) of bisphosphoramide $(R, R)-3,123 \mu \mathrm{L}(1.1 \mathrm{mmol}$, 1.1 equiv) of $\mathrm{SiCl}_{4}$, and $293 \mathrm{mg}(1.2 \mathrm{mmol}, 1.2$ equiv) of (E)-1-[(tert-butoxy)-propenyl]-tertbutyldimethylsilane $((E)-5 d)$ to yield, after Kugelrohr distillation, $280 \mathrm{mg}(98 \%)$ of $(2 S, 3 R)-\mathbf{6 d b}$ as a colorless wax.

Data for $(2 S, 3 R)-\mathbf{6 d \mathbf { d b }}$ :

bp: $\quad 210^{\circ} \mathrm{C}(0.01 \mathrm{mmHg}, \mathrm{ABT})$

${ }^{1} \mathrm{H}$ NMR: $\quad\left(500 \mathrm{MHz}, \mathrm{CDCl}_{3}\right)$

$8.2(\mathrm{~d}, J=8.1,1 \mathrm{H}, \mathrm{HC}(11)), 7.88$ (d, $J=7.9,1 \mathrm{H}, \mathrm{HC}(14)), 7.8(\mathrm{~d}, J=8.35,1 \mathrm{H}$, $\mathrm{HC}(8)), 7.57$ (d, $J=7.5,1 \mathrm{H}, \mathrm{HC}(6))$, 7.51-7.45 (m, 3 H, HC(7,12,13)), 5.48 (dd, $J=7.5,5.14,1 \mathrm{H}, \mathrm{HC}(3)), 3.52(\mathrm{~d}, J=5.14,1 \mathrm{H}, \mathrm{OH}), 3.04$ (pent, $J=7.3,1 \mathrm{H}$, $\mathrm{HC}(2)), 1.44$ (s, $\left.9 \mathrm{H}, \mathrm{H}_{3} \mathrm{C}(16)\right), 1.06$ (s, $\left.3 \mathrm{H}, \mathrm{H}_{3} \mathrm{C}(4)\right)$

${ }^{13} \mathrm{C} \mathrm{NMR}: \quad\left(125 \mathrm{MHz}, \mathrm{CDCl}_{3}\right)$

175.59 (C(1)), 137.44 (C(5)), 133.82 (C(9)), 130.91 (C(10)), 128.88 (C(14)), 128.39 (C(8)), 126.02 (C(12)), 125.49 (C(13)), 125.23 (C(7)), 124.51 (C(6)), 123.51 (C(11)), 81.29 (C(15)), 73.57 (C(3)), 47.05 (C(2)), 28 (C(16)), 15.29 (C(4))

IR: $\quad\left(\mathrm{CHCl}_{3}\right)$

3606 (w), 3474 (w), 2981 (m), 1712 (s), 1369 (s), 1154 (s), 780 (m)

MS: $\quad(\mathrm{EI}, 70 \mathrm{eV})$

$286\left(\mathrm{M}^{+}, 8\right), 230(5), 213(9), 151$ (100), $129(50), 73$ (12)

Opt. Rot.: $\quad[\alpha]_{\mathrm{D}}^{24}+28.61 \quad(\mathrm{c}=1.0, \mathrm{EtOH})$ 
TLC: $\quad R_{f} 0.5$ (hexanes/EtOAc, 8/2) [UV(254)/ $\left.\mathrm{KMnO}_{4}\right]$

SFC: $\quad t_{R}(2 R, 3 S)-6 \mathbf{d b}, 4.11(2.8 \%) ; t_{R}(2 S, 3 R)-6 \mathbf{d b}, 4.52 \mathrm{~min}(97.2 \%)$ (Chiralpak AD, 150 bar, $40{ }^{\circ} \mathrm{C}, 5.0 \% \mathrm{CH}_{3} \mathrm{OH}$ in $\mathrm{CO}_{2}, 3.0 \mathrm{~mL} / \mathrm{min}, 220 \mathrm{~nm}$ )

Analysis: $\quad \mathrm{C}_{18} \mathrm{H}_{22} \mathrm{O}_{3}(286.37)$
Calcd:
C, 75.50;
$\mathrm{H}, 7.74 \%$
Found:
C, 75.57;
$\mathrm{H}, 7.90 \%$

\section{Preparation of tert-Butyl (2S,3R)-3-Hydroxy-2-methyl-3-(2-naphthalenyl)propanoate $((2 S, 3 R)-6 \mathrm{dc})$}

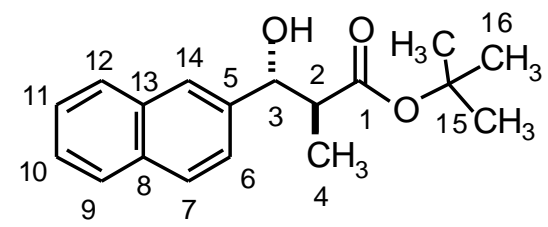

Following General Procedure 2, $156 \mathrm{mg}$ (1.0 mmol, 1.0 equiv.) of 2-naphthaldehyde was combined with $8.4 \mathrm{mg}(0.01 \mathrm{mmol}, 0.01$ equiv) of bisphosphoramide $(R, R)-3,123 \mu \mathrm{L}(1.1 \mathrm{mmol}$, 1.1 equiv) of $\mathrm{SiCl}_{4}$, and $293 \mathrm{mg}(1.2 \mathrm{mmol}, 1.2$ equiv) of (E)-1-[(tert-butoxy)-propenyl]-tertbutyldimethylsilane $((E)-5 d)$ to yield, after Kugelrohr distillation, $271 \mathrm{mg}(95 \%)$ of $(2 S, 3 R)$-6dc as a colorless wax.

Data for $(2 S, 3 R)-\mathbf{6 d c}$ :

bp: $\quad 210^{\circ} \mathrm{C}(0.01 \mathrm{mmHg}, \mathrm{ABT})$

${ }^{1} \mathrm{H}$ NMR: $\quad\left(500 \mathrm{MHz}, \mathrm{CDCl}_{3}\right)$

7.83 (m, 4 H, H(Ar)), 7.48 (m, 3 H, H(Ar)), 4.87 (d, J = 8.14, 1 H, HC(3)), 3.33

(br s, $1 \mathrm{H}, \mathrm{OH}), 2.82$ (pent, $J=7.28,1 \mathrm{H}, \mathrm{HC}(2)), 1.44$ (s, $\left.9 \mathrm{H}, \mathrm{H}_{3} \mathrm{C}(16)\right), 1.04$ (d, $\left.J=7.28,3 \mathrm{H}, \mathrm{H}_{3} \mathrm{C}(4)\right)$

${ }^{13} \mathrm{C} \mathrm{NMR}: \quad\left(125 \mathrm{MHz}, \mathrm{CDCl}_{3}\right)$

175.3 (C(1)), 139.2 (C(5)), 133.13 (C(13)), 133.10 (C(8)), 128.2 (C(9)), 127.9 (C(12)), 127.6 (C96)), 126.1 (C(7)), 125.9 (C(14)), 125.8 (C(11)), 124.3 (C(10)), 81.27 (C(15)), $76.5(\mathrm{C}(3)), 47.89(\mathrm{C}(2)), 27.98$ (C(16)), $15.72(\mathrm{C}(4))$

IR: (neat)

3343 (w), 2977 (s), 2935 (m), 1724 (s), 1711 (s), 1457 (s), 1368 (s), 1255 (s), 1155 (s), 1026 (s), $821(\mathrm{~m}), 748(\mathrm{~m})$ 
MS: $\quad(\mathrm{EI}, 70 \mathrm{eV})$

$286\left(\mathrm{M}^{+}, 8\right), 229$ (10), 193 (10), 151 (100), 129 (51), 73 (12)

Opt. Rot.: $\quad[\alpha]_{\mathrm{D}}^{24}+21.37(\mathrm{c}=1, \mathrm{EtOH})$

TLC: $\quad R_{f} 0.44$ (hexanes/EtOAc, 8/2) $\left[\mathrm{UV}(254) / \mathrm{KMnO}_{4}\right]$

SFC: $\quad t_{R}(2 S, 3 R)-\mathbf{6 d c}, 3.26(99.6 \%) ; t_{R}(2 R, 3 S)$-6dc, $3.67 \mathrm{~min}(0.4 \%)$ (Chiralpak AS, 150 bar, $40{ }^{\circ} \mathrm{C}, 5.0 \% \mathrm{CH}_{3} \mathrm{OH}$ in $\mathrm{CO}_{2}, 2.5 \mathrm{~mL} / \mathrm{min}, 220 \mathrm{~nm}$ )

Analysis: $\quad \mathrm{C}_{18} \mathrm{H}_{22} \mathrm{O}_{3}(286.37)$
Calcd:
C, 75.50;
$\mathrm{H}, 7.74 \%$

Found:

C, 75.41;

$\mathrm{H}, 7.78 \%$

Preparation of tert-Butyl (2S,3R)-3-Hydroxy-3-(4-methoxyphenyl)-2-methylpropanoate $((2 S, 3 R)-6 \mathrm{de})$

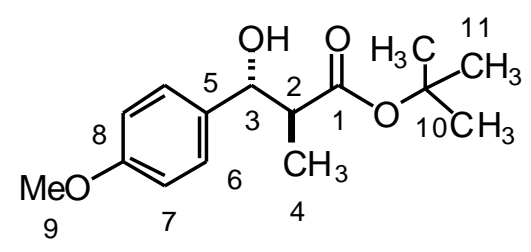

Following General Procedure 2, $122 \mu \mathrm{L}(1.0 \mathrm{mmol}, 1.0$ equiv) of $p$-anisaldehyde was combined with $8.4 \mathrm{mg}$ (0.01 mmol, 0.01 equiv.) of bisphosphoramide $(R, R)-\mathbf{3}, 123 \mu \mathrm{L}(1.1$ mmol, 1.1 equiv) of $\mathrm{SiCl}_{4}$, and $293 \mathrm{mg}$ (1.2 mmol, 1.2 equiv) of (E)-1-[(tert-butoxy)-propenyl]tert-butyldimethylsilane ((E)-5d) to yield, after Kugelrohr distillation, $233 \mathrm{mg}(88 \%)$ of $(2 S, 3 R)$ 6de as a colorless oil.

Data for $(2 S, 3 R)$-6de:

bp: $\quad 175^{\circ} \mathrm{C}(0.01 \mathrm{mmHg}, \mathrm{ABT})$

1H NMR: $\quad\left(500 \mathrm{MHz}, \mathrm{CDCl}_{3}\right)$

7.16 (m, 2 H, HC(6)), 6.77 (m, 2 H, HC(7)), 4.55 (d, $J=8.36,1$ H, HC(3)), 2.99 (s, $1 \mathrm{H}, \mathrm{OH}$ ), 2.57 (pent, $J=7.28,1 \mathrm{H}, \mathrm{HC}(2)), 1.35$ (s, $9 \mathrm{H}, \mathrm{H}_{3} \mathrm{C}(11)$ ), 0.88 (d, $J$ $\left.=7.28,3 \mathrm{H}, \mathrm{H}_{3} \mathrm{C}(4)\right)$

${ }^{13} \mathrm{C} \mathrm{NMR}: \quad\left(125 \mathrm{MHz}, \mathrm{CDCl}_{3}\right)$

$175.43(\mathrm{C}(1)), 159.2(\mathrm{C}(8)), 133.98(\mathrm{C}(5)), 127.84(\mathrm{C}(6)), 113.72(\mathrm{C}(7)), 81.08$ (C(10)), $75.93(\mathrm{C}(3)), 55.23(\mathrm{C}(9)), 47.81(\mathrm{C}(2)), 27.99(\mathrm{C}(11)), 14.60(\mathrm{C}(4))$ 
IR: (neat)

3459 (w), 2977 (m), 29369 (m), 1726 (s), 1612 (m), 1513 (s), 1460 (m), 1368 (s), 1251 (s), 1154 (s), 1035 (m), 833 (m)

MS.: (EI, $70 \mathrm{eV})$

$$
266\left(\mathrm{M}^{+}, 8\right), 209(3), 193 \text { (10), } 134 \text { (100), } 108 \text { (9), } 76 \text { (7) }
$$

Opt. Rot.: $[\alpha]_{\mathrm{D}}^{24}+24.59(\mathrm{c}=1, \mathrm{EtOH})$

TLC: $\quad R_{f} 0.34$ (hexanes/EtOAc, 8/2) [UV(254)/KMnO $\mathrm{KM}_{4}$

SFC: $\quad t_{R}(2 S, 3 R)$-6de, $2.46(99.2 \%) ; t_{R}(2 R, 3 S)$-6de, 3.05 min $(0.8 \%)$ (Chiralpak AS, 150 bar, $40{ }^{\circ} \mathrm{C}, 5.0 \% \mathrm{CH}_{3} \mathrm{OH}$ in $\mathrm{CO}_{2}, 2.5 \mathrm{~mL} / \mathrm{min}, 220 \mathrm{~nm}$ )

Analysis: $\quad \mathrm{C}_{15} \mathrm{H}_{22} \mathrm{O}_{4}(266.33)$
Calcd:
C, 67.64;
$\mathrm{H}, 8.33 \%$
Found:
C, 67.58;
$\mathrm{H}, 8.44 \%$

Preparation of tert-Butyl (2S,3R)-3-Hydroxy-2-methyl-3-(4-trifluoromethylphenyl)propanoate $((2 S, 3 R)-6 \mathrm{df})$

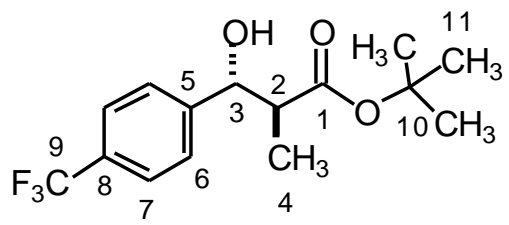

Following General Procedure 2, $136 \mu \mathrm{L} \quad(1.0 \quad \mathrm{mmol}, \quad 1.0$ equiv $)$ of 4trifluoromethylbenzaldehyde was combined with $8.4 \mathrm{mg}(0.01 \mathrm{mmol}, 0.01$ equiv $)$ of bisphosphoramide $(R, R)-3,123 \mu \mathrm{L}$ (1.1 mmol, 1.1 equiv) of $\mathrm{SiCl}_{4}$, and $293 \mathrm{mg}$ (1.2 mmol, 1.2 equiv) of (E)-1-[(tert-butoxy)-propenyl]-tert-butyldimethylsilane $((E)-5 \mathbf{d})$ to yield, after Kugelrohr distillation, $284 \mathrm{mg}(93 \%)$ of $(2 S, 3 R)-\mathbf{6 d f}$ as a white crystalline solid.

Data for $(2 S, 3 R)-\mathbf{6 d f}$ :

mp: $\quad 84-86{ }^{\circ} \mathrm{C}$ (hexane)

${ }^{1} \mathrm{H}$ NMR: $\quad\left(400 \mathrm{MHz}, \mathrm{CDCl}_{3}\right)$

7.53 (m, $4 \mathrm{H}, \mathrm{HC}(6,7)), 4.57$ (d, $J=7.56,1 \mathrm{H}, \mathrm{HC}(3)), 3.48$ (s, $1 \mathrm{H}, \mathrm{OH}), 2.69$ (pent, $J=7.32,1 \mathrm{H}, \mathrm{HC}(2)), 1.41\left(\mathrm{~s}, 9 \mathrm{H}, \mathrm{H}_{3} \mathrm{C}(11)\right), 1.06(\mathrm{~d}, J=7.08,3 \mathrm{H}$, $\left.\mathrm{H}_{3} \mathrm{C}(4)\right)$ 
${ }^{13} \mathrm{C}$ NMR: $\quad\left(100 \mathrm{MHz}, \mathrm{CDCl}_{3}\right)$

175.24 (C(1)), $146.20(\mathrm{C}(5)), 130.30$ (q, $J=32.04, \mathrm{C}(8)), 127.17$ (C(6)), 125.52

(C(7)), $14.34(\mathrm{q}, J=271.6, \mathrm{C}(9)), 81.63$ (C(10)), 75.60 (C(3)), 47.38 (C(2)), $27.93(\mathrm{C}(11)), 14.63(\mathrm{C}(4))$

F NMR: $\quad\left(376 \mathrm{MHz}, \mathrm{CDCl}_{3}\right)$

$-62.89\left(\mathrm{t}, 3 \mathrm{~F}, \mathrm{~F}_{3} \mathrm{C}(9)\right)$

IR: $\quad\left(\mathrm{CHCl}_{3}\right)$

3492 (w), 3009 (w), 2982 9w), 2938 (w), 1707 (s), 1369 (m), 1166 (s), 1131 (s) $1068(\mathrm{~m}), 8449$ (m)

MS.: (EI, $70 \mathrm{eV})$

305 (2), 248 (13), 229 (16), 175 (60), 130 (32), 73 (59)

Opt. Rot.: $\quad[\alpha]_{\mathrm{D}}^{24}+39.50(\mathrm{c}=1, \mathrm{EtOH})$

TLC: $\quad R_{f} 0.48$ (hexanes/EtOAc, 8/2) [UV(254)/KMnO $\left.{ }_{4}\right]$

SFC: $\quad t_{R}(2 S, 3 R)-6 \mathbf{d f}, 2.44(96.0 \%) ; t_{R}(2 R, 3 S)-6 \mathbf{d f}, 3.08 \mathrm{~min}(4.0 \%)$ (Whelk-O1, 150 bar, $40{ }^{\circ} \mathrm{C}, 1.0 \% \mathrm{CH}_{3} \mathrm{OH}$ in $\mathrm{CO}_{2}, 3.5 \mathrm{~mL} / \mathrm{min}, 220 \mathrm{~nm}$ )

Analysis: $\quad \mathrm{C}_{15} \mathrm{H}_{19} \mathrm{~F}_{3} \mathrm{O}_{3}(248.20)$
Calcd:
C, 59.20;
H, 6.29;
F, $18.73 \%$
Found:
C, 59.05;
H, 6.21;
F, $18.61 \%$

Preparation of tert-Butyl $(2 S, 3 S)-3-H y d r o x y-2-m e t h y l-5-p h e n y l-4-p e n t e n o a t e ~((2 S, 3 S)-6 \mathrm{dg})$

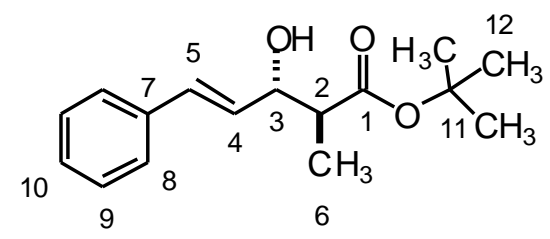

Following General Procedure 2, $141 \mu \mathrm{L}$ (1.0 mmol, 1.0 equiv) of cinnamaldehyde was combined with $8.4 \mathrm{mg}(0.01 \mathrm{mmol}, 0.01$ equiv $)$ of bisphosphoramide $(R, R)-3,123 \mu \mathrm{L}(1.1 \mathrm{mmol}$, 1.1 equiv) of $\mathrm{SiCl}_{4}$, and $293 \mathrm{mg}(1.2 \mathrm{mmol}, 1.2$ equiv) of (E)-1-[(tert-butoxy)-propenyl]-tertbutyldimethylsilane (5d) to yield, after Kugelrohr distillation, $256 \mathrm{mg}(98 \%)$ of $(2 S, 3 S)-\mathbf{6 d g}$ as a white crystalline solid. 


\section{Data for $(2 S, 3 S)-\mathbf{6 d g}$ :}

mp: $\quad 80-82^{\circ} \mathrm{C}$ (hexane)

${ }^{1} \mathrm{H}$ NMR: $\quad\left(400 \mathrm{MHz}, \mathrm{CDCl}_{3}\right)$

$7.15(\mathrm{~m}, 5 \mathrm{H}, \mathrm{HC}(8,9,10)), 6.64$ (d, $J=15.87,1 \mathrm{H}, \mathrm{HC}(5)), 6.16(\mathrm{dd}, J=15.86$, 6.59, $1 \mathrm{H}, \mathrm{HC}(4)), 4.33(\mathrm{t}, J=6.59,1 \mathrm{H}, \mathrm{HC}(3)), 2.55$ (pent, $J=7.08,1 \mathrm{H}$, $\mathrm{HC}(2)), 1.46$ (s, $\left.9 \mathrm{H}, \mathrm{H}_{3} \mathrm{C}(12)\right), 1.20$ (d, $\left.J=7.32,3 \mathrm{H}, \mathrm{H}_{3} \mathrm{C}(6)\right)$

${ }^{13} \mathrm{C} \mathrm{NMR}: \quad\left(100 \mathrm{MHz}, \mathrm{CDCl}_{3}\right)$

174.97 (C(1)), 136.50 (C(7)), 131.73 (C(5)), 129.53 (C(4)), 128.55 (C(8)), 127.73

(C(10)), 126.50 (C(9)), 81.24 (C(11)), 74.62 (C(3)), 46.19 (C(2)), 28.05 (C(12)), $14.25(\mathrm{C}(6))$

IR: (neat)

3521 (w), 3011 (m), 2982 (m), 2937 (m), 1718 (s), 1457 (w), 1369 (m), 1154 (s), $968(\mathrm{~m})$

MS.: $\quad(\mathrm{EI}, 70 \mathrm{eV})$

262 (3), 245 (20), 206 (37), 189 (99), 143 (25), 133 9100), 105 (16), 57 (86)

Opt. Rot.: $\quad[\alpha]_{\mathrm{D}}^{24}-17.48(\mathrm{c}=1, \mathrm{EtOH})$

TLC: $\quad R_{f} 0.36$ (hexanes/EtOAc, 8/2) [UV(254)/ $\left./ \mathrm{KMnO}_{4}\right]$

SFC: $\quad t_{R}(2 S, 3 S)-\mathbf{6 d g}, 3.31(>99.0 \%) ; t_{R}(2 R, 3 R)-\mathbf{6 d g}, 4.13 \mathrm{~min}(<1.0 \%)$ (Chiralpak AS, 150 bar, $40{ }^{\circ} \mathrm{C}, 1.0 \% \mathrm{CH}_{3} \mathrm{OH}$ in $\mathrm{CO}_{2}, 3.5 \mathrm{~mL} / \mathrm{min}, 220 \mathrm{~nm}$ )

Analysis: $\quad \mathrm{C}_{16} \mathrm{H}_{22} \mathrm{O}_{3}(262.34)$
Calcd:
C, 73.25;
$\mathrm{H}, 8.45 \%$
Found:
C, 73.18;
$\mathrm{H}, 8.45 \%$ 
Preparation of tert-Butyl (2S,3R)-3-Hydroxy-2,4-dimethyl-5-phenyl-4-pentenoate ((2S,3R)6dh)

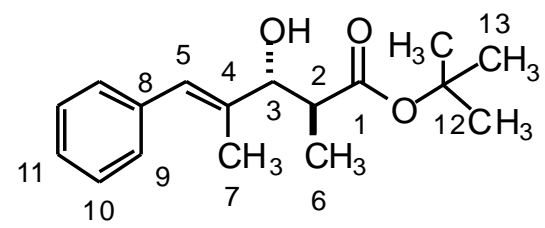

Following General Procedure 2, $140 \mu \mathrm{L} \quad(1.0 \mathrm{mmol}, \quad 1.0$ equiv $)$ of $\alpha-$ methylcinnamaldehyde was combined with $8.4 \mathrm{mg} \quad(0.01 \mathrm{mmol}, \quad 0.01$ equiv $)$ of bisphosphoramide $(R, R)-3,123 \mu \mathrm{L}$ (1.1 mmol, 1.1 equiv) of $\mathrm{SiCl}_{4}$, and $293 \mathrm{mg}$ (1.2 mmol, 1.2 equiv) of (E)-1-[(tert-butoxy)-propenyl]-tert-butyldimethylsilane $((E)$-5d) to yield, after Kugelrohr distillation, $248 \mathrm{mg}(90 \%)$ of $(2 S, 3 R)-\mathbf{6 d h}$ as a colorless wax.

\section{Data for $(2 S, 3 R)-\mathbf{6 d h}$ :}

bp: $\quad 225^{\circ} \mathrm{C}(0.01 \mathrm{mmHg}, \mathrm{ABT})$

ㅁN NMR: $\quad\left(400 \mathrm{MHz}, \mathrm{CDCl}_{3}\right)$

7.25 (m, 5 H, HC(9,10,11)), 6.53 (s, $1 \mathrm{H}, \mathrm{HC}(5)), 4.20$ (d, J = 7.81, $1 \mathrm{H}, \mathrm{HC}(3))$, 3.00 (s, $1 \mathrm{H}, \mathrm{OH}$ ), 2.67 (pent, $J=7.81,1 \mathrm{H}, \mathrm{HC}(2)), 1.87$ (s, $3 \mathrm{H}, \mathrm{H}_{3} \mathrm{C}(7)$ ), 1.47 (s, $\left.9 \mathrm{H}, \mathrm{H}_{3} \mathrm{C}(13)\right), 1.13\left(\mathrm{~d}, J=7.32,3 \mathrm{H}, \mathrm{H}_{3} \mathrm{C}(6)\right)$

${ }^{13} \mathrm{C} \mathrm{NMR}: \quad\left(100 \mathrm{MHz}, \mathrm{CDCl}_{3}\right)$

$175.28(\mathrm{C}(1)), 137.33$ (C(4)), $137.24(\mathrm{C}(8)), 128.98$ (C(10)), 128.07 (C(11)), 127.83 (C(9)), 126.54 (C(5)), 81.22 (C(12)), 79.88 (C(3)), 43.92 (C(2)), 28.04 $(\mathrm{C}(12)), 14.67(\mathrm{C}(7)), 13.10(\mathrm{C}(6))$

IR: $\quad\left(\mathrm{CHCl}_{3}\right)$

3454 (w), 2977 (s), 2935 (m), 1726 (s), 1454 (m), 1368 (m), 1254 (m), 1154 (s), $1009(\mathrm{~m}), 649(\mathrm{~m})$

MS: $\quad(\mathrm{EI}, 70 \mathrm{eV})$

277 (M+, 2), 261 (2), 220 (19), 203 (43), 157 (43), 157 (24), 147 (100), 129 (27), $91(18), 57(38)$

Opt. Rot.: $\quad[\alpha]_{\mathrm{D}}^{24}-66.28(\mathrm{c}=1.0, \mathrm{EtOH})$

TLC: $\quad R_{f} 0.39$ (hexanes/EtOAc, 8/2) [UV(254)/ $\left.\mathrm{KMnO}_{4}\right]$ 
SFC: $\quad t_{R}(2 S, 3 R)-6 \mathbf{d h}, 3.63(96.1 \%) ; t_{R}(2 R, 3 S)-6 \mathbf{d h}, 4.13 \mathrm{~min}(3.9 \%)$ (Chiralpak AS, 150 bar, $40{ }^{\circ} \mathrm{C}, 1.0 \% \mathrm{CH}_{3} \mathrm{OH}$ in $\mathrm{CO}_{2}, 3.0 \mathrm{~mL} / \mathrm{min}, 220 \mathrm{~nm}$ )

Analysis: $\quad \mathrm{C}_{17} \mathrm{H}_{24} \mathrm{O}_{3}(276.38)$
Calcd:
C, 73.88;
$\mathrm{H}, 8.75 \%$
Found:
C, 73.59;
$\mathrm{H}, 8.63 \%$

Preparation of tert-Butyl (2S,3R)-3-Hydroxy-2-methyl-5-phenyl-4-pentynoate $((2 S, 3 R)-6 \mathrm{dl})$

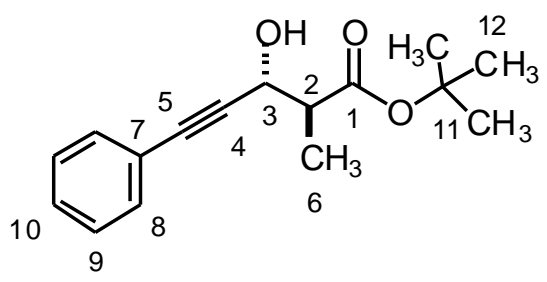

Following General Procedure 2, $122 \mu \mathrm{L}$ (1.0 mmol, 1.0 equiv) of 3-phenyl-2-propynal was combined with $8.4 \mathrm{mg}(0.01 \mathrm{mmol}, 0.01$ equiv) of bisphosphoramide $(R, R)-3,123 \mu \mathrm{L}(1.1$ mmol, 1.1 equiv) of $\mathrm{SiCl}_{4}$, and $293 \mathrm{mg}(1.2 \mathrm{mmol}, 1.2$ equiv) of (E)-1-[(tert-butoxy)-propenyl]tert-butyldimethylsilane $((E)-5 d)$ to yield, after Kugelrohr distillation, $240 \mathrm{mg}(92 \%)$ of $(2 S, 3 R)$ 6dl as a white crystalline solid.

Data for $(2 S, 3 R)-\mathbf{6 d l}$ :

bp: $\quad 210^{\circ} \mathrm{C}(0.01 \mathrm{mmHg}, \mathrm{ABT})$

mp: $\quad 68-70{ }^{\circ} \mathrm{C}$ (hexane)

${ }^{1} \mathrm{H}$ NMR: $\quad\left(500 \mathrm{MHz}, \mathrm{CDCl}_{3}\right)$

7.42 (m, 2 H, HC(8)), 7.30 (m, 3 H, HC(9,10)), 4.67 (t, J = 7.07, $1 \mathrm{H}, \mathrm{HC}(3))$, 3.09 (d, $J=7.07,1 \mathrm{H}, \mathrm{OH}), 2.73$ (pent, $J=7.07,1 \mathrm{H}, \mathrm{HC}(2)), 1.48$ (s, $9 \mathrm{H}$, $\left.\mathrm{H}_{3} \mathrm{C}(12)\right), 1.32$ (d, $\left.J=7.29,3 \mathrm{H}, \mathrm{H}_{3} \mathrm{C}(6)\right)$

${ }^{13} \mathrm{C} \mathrm{NMR}: \quad\left(125 \mathrm{MHz}, \mathrm{CDCl}_{3}\right)$

174.10 (C(1)), 131.71 (C(4)), 128.48 (C(10)), 128.27 (C(8)), 122.45 (C(7)), 87.96 (C(6)), 85.54 (C(7)), 81.46 (C911)), 64.85 (C93)), 46.95 (C(2)), 28.02 (C(12)), $13.95(\mathrm{C}(6))$ 
IR: $\quad\left(\mathrm{CHCl}_{3}\right)$

3485 (w), 3028 (w), 3010 (m), 2982 (m), 2938 (m), 1712 (s), 1600 (w), 1490 (w), $1458(\mathrm{w}), 1369$ (m), 1253 (m), 1155 (s), $1028(\mathrm{w})$

MS: $\quad(\mathrm{EI}, 70 \mathrm{eV})$

$261\left(\mathrm{M}^{+}, 23\right), 243(8), 205$ (44), 187 (100), 131 (39)

Opt. Rot.: $\quad[\alpha]_{\mathrm{D}}^{24}+6.87(\mathrm{c}=1.0, \mathrm{EtOH})$

TLC: $\quad R_{f} 0.41$ (hexanes/EtOAc, 8/2) [UV(254)/KMnO $\mathrm{KM}_{4}$

SFC: $\quad t_{R}(2 S, 3 R)-6 \mathbf{d l}, 4.78(84.5 \%) ; t_{R}(2 R, 3 S)-6 \mathbf{d l}, 5.71 \mathrm{~min}(15.5 \%)$ (Chiralpak AS, 150 bar, $40{ }^{\circ} \mathrm{C}, 1.5 \% \mathrm{CH}_{3} \mathrm{OH}$ in $\mathrm{CO}_{2}, 2.5 \mathrm{~mL} / \mathrm{min}, 220 \mathrm{~nm}$ )

Analysis: $\quad \mathrm{C}_{16} \mathrm{H}_{20} \mathrm{O}_{3}(260.33)$

Calcd: $\quad$ C, $73.82 ; \quad$ H, $7.74 \%$

Found: $\quad$ C, $73.92 ; \quad H, 7.76 \%$

Preparation of Ethyl (2S,3S)-3-Hydroxy-2-methyl-5-phenylpentanoate ((2S,3S)-6bk)

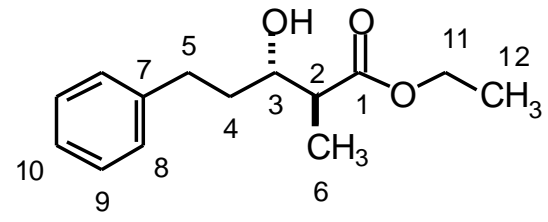

A flame-dried, 10-mL, 2-neck round-bottom flask containing a solution of $42.1 \mathrm{mg}(0.05$ mmol, 0.05 equiv) of bisphosphoramide $(R, R)-3$ and $37 \mathrm{mg}(0.1 \mathrm{mmol}, 0.1$ equiv) of tetrabutylammonium iodide in $\mathrm{CH}_{2} \mathrm{Cl}_{2}(2.5 \mathrm{~mL})$ was cooled to $-78{ }^{\circ} \mathrm{C}$ under nitrogen. Into this mixture was added $123 \mu \mathrm{L}(1.1 \mathrm{mmol}, 1.1$ equiv $)$ of $\mathrm{SiCl}_{4}$. To the resulting mixture was added $132 \mu \mathrm{L}$ (1.0 mmol, 1.0 equiv) of hydrocinnamaldehyde and it was allowed to stir at $-78{ }^{\circ} \mathrm{C}$ for 5 min. Then $260 \mathrm{mg}$ (1.2 mmol, 1.2 equiv) of (E)-[(1-ethoxy)-propenyl]-tert-butyldimethylsilane $((E)-5 \mathbf{b})$ was added dropwise (over $5 \mathrm{~min}$ ) to the reaction mixture. The resulting mixture was allowed to stir at $-78{ }^{\circ} \mathrm{C}$ (bath temperature) for $24 \mathrm{~h}$ whereupon the cold reaction mixture was poured into a rapidly stirring $1 / 1$ sat. aq. $\mathrm{KF} / 1.0 \mathrm{M} \mathrm{KH}_{2} \mathrm{PO}_{4}$ solution $(20 \mathrm{~mL})$. This biphasic mixture was stirred vigorously for $1 \mathrm{~h}$ before filtration through Celite. The aqueous layer was washed with $\mathrm{CH}_{2} \mathrm{Cl}_{2}(3 \times 50 \mathrm{~mL})$. The combined organic extracts were washed with brine (50 $\mathrm{mL})$, dried over $\mathrm{Na}_{2} \mathrm{SO}_{4}(2 \mathrm{~g})$, filtered and the filtrate was concentrated in vacuo. The residue 
was purified by column chromatography (hexanes/EtOAc, 9/1), to yield $167 \mathrm{mg}$ (71\%) of $(2 S, 3 S)-\mathbf{6 b k}$ as a colorless oil.

\section{Data for $(2 S, 3 S)$-6bk:}

${ }^{1} \mathrm{H}$ NMR: $\quad\left(400 \mathrm{MHz}, \mathrm{CDCl}_{3}\right)$

7.20 (m, 5 H, HC(8,9,10)), 4.18 (q, J = 7.08, 2 H, HC(11)), 3.68 (m, $1 \mathrm{H}, \mathrm{HC}(3))$,

2.89 (m, $1 \mathrm{H}, \mathrm{HC}(5)), 2.77$ (d, $J=7.08,1 \mathrm{H}, \mathrm{OH}), 2.67$ (m, $1 \mathrm{H}, \mathrm{HC}(5)), 2.53$

(pent, $J=6.34,1 \mathrm{H}, \mathrm{HC}(2)), 1.80(\mathrm{~m}, 2 \mathrm{H}, \mathrm{HC}(4)), 1.28(\mathrm{t}, J=7.08,3 \mathrm{H}$, $\left.\mathrm{H}_{3} \mathrm{C}(12)\right), 1.22\left(\mathrm{~d}, J=7.32,3 \mathrm{H}, \mathrm{H}_{3} \mathrm{C}(6)\right)$

${ }^{13} \mathrm{C} \mathrm{NMR}: \quad\left(100 \mathrm{MHz}, \mathrm{CDCl}_{3}\right)$

176.1 (C(1)), $141.94(\mathrm{C}(9)), 128.46$ (C(10)), 128.39 (C(8)), 125.85 (C(7)), 72.63

(C(11)), 60.64 (C(3)), 45.15 (C(2)), 36.67 (C(5)), 31.91 (C(4)), 14.41 (C(12)), $14.17(\mathrm{C}(6))$

IR: (neat)

3459 (m), 3026 (w), 2980 (s), 2940 (s), 1726 (s), 1495 (w), 1455 (s), 1376 (s),

1262 (s), 1187 (s), 1096 (s), 1043 (s)

MS: $\quad(\mathrm{EI}, 70 \mathrm{eV})$

$237\left(\mathrm{M}^{+}, 53\right), 219$ (52), 173 (19), 144 (65), 117 (100), 91 (73)

Opt. Rot.: $\quad[\alpha]_{\mathrm{D}}^{24}-14.87(\mathrm{c}=1.0, \mathrm{EtOH})$

TLC: $\quad R_{f} 0.1$ (hexanes/EtOAc, 9/1) [UV(254)/KMnO $\left.{ }_{4}\right]$

SFC: $\quad t_{R}(2 S, 3 S)-6 \mathbf{b k}, 4.34(94.9 \%) ; t_{R}(2 R, 3 R)-6 \mathbf{b k}, 5.04 \mathrm{~min}(5.2 \%)$ (Chiralpak OD, 150 bar, $40{ }^{\circ} \mathrm{C}, 2.0 \% \mathrm{CH}_{3} \mathrm{OH}$ in $\mathrm{CO}_{2}, 3.5 \mathrm{~mL} / \mathrm{min}, 220 \mathrm{~nm}$ )

Analysis: $\quad \mathrm{C}_{14} \mathrm{H}_{20} \mathrm{O}_{3}(236.31)$
Calcd:
C, 71.16;
$\mathrm{H}, 8.53 \%$
Found:
C, 70.95;
H, $8.52 \%$ 


\section{Preparation of Ethyl (2S,3S)-3-Cyclohexyl-3-hydroxy-2-methylpropanoate ((2S,3S)-6bj)}

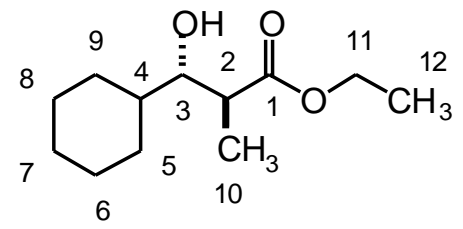

A flame-dried, 10-mL, 2-neck, round-bottom flask containing a solution of $84.2 \mathrm{mg}(0.1$ mmol, 0.1 equiv) of bisphosphoramide $(R, R)-3$ and $37 \mathrm{mg}(0.1 \mathrm{mmol}, 0.1$ equiv) of tetrabutylammonium iodide in $\mathrm{CH}_{2} \mathrm{Cl}_{2}(1.25 \mathrm{~mL})$ was cooled to $-40{ }^{\circ} \mathrm{C}$ under nitrogen. Into this mixture was added $123 \mu \mathrm{L}\left(1.1 \mathrm{mmol}, 1.1\right.$ equiv) of $\mathrm{SiCl}_{4}$. To the resulting mixture was added $121 \mu \mathrm{L}$ (1.0 mmol, 1.0 equiv) of cyclohexane carboxaldehyde and it was allowed to stir at $-78^{\circ} \mathrm{C}$ for $5 \mathrm{~min}$. Then $260 \mathrm{mg}(1.2 \mathrm{mmol}, 1.2 \mathrm{equiv})$ of (E)-[(1-ethoxy)-propenyl]-tertbutyldimethylsilane $((E)-5 \mathbf{b})$ was added dropwise (over $5 \mathrm{~min}$ ) to the reaction mixture. The resulting mixture was allowed to stir at $-40{ }^{\circ} \mathrm{C}$ (bath temperature) for $24 \mathrm{~h}$ whereupon the cold reaction mixture was poured into a rapidly stirring $1 / 1$ sat. aq. $\mathrm{KF} / 1.0 \mathrm{M} \mathrm{K \textrm {KH } _ { 2 }} \mathrm{PO}_{4}$ solution (20 $\mathrm{mL}$ ). This biphasic mixture was stirred vigorously for $1 \mathrm{~h}$ before filtration through Celite. The aqueous layer was washed with $\mathrm{CH}_{2} \mathrm{Cl}_{2}(3 \times 50 \mathrm{~mL})$. The combined organic extracts were washed with brine $(50 \mathrm{~mL})$, dried over $\mathrm{Na}_{2} \mathrm{SO}_{4}(2 \mathrm{~g})$, filtered and the filtrate was concentrated in vасио. The residue was purified by column chromatography (hexanes/EtOAc, 9/1), to yield 86 mg $(40 \%)$ of $(2 S, 3 S)-\mathbf{6 b j}$ as a colorless oil.

\section{Data for $(2 S, 3 S)-\mathbf{6 b j}$ :}

글 $\quad\left(400 \mathrm{MHz}, \mathrm{CDCl}_{3}\right)$

4.17 (m, $2 \mathrm{H}, \mathrm{HC}(11)), 3.35$ (q, $J=6.59,1 \mathrm{H}, \mathrm{HC}(3)$ ), 2.65 (pent, $J=5.85,1 \mathrm{H}$, $\mathrm{HC}(2)), 2.57$ (d, $J=7.81,1 \mathrm{H}, \mathrm{OH}), 1.85-1.65$ (m, $4 \mathrm{H}, \mathrm{HC}(6,8)), 1.6-1.5$ (m, $4 \mathrm{H}$, $\mathrm{HC}(5,9)), 1.27$ (t, $\left.J=7.08,3 \mathrm{H}, \mathrm{H}_{3} \mathrm{C}(12)\right), 1.22$ (d, $\left.J=7.08,3 \mathrm{H}, \mathrm{H}_{3} \mathrm{C}(10)\right), 1.2-$ $1.0(\mathrm{~m}, 3 \mathrm{H}, \mathrm{HC}(4,7))$

${ }^{13} \mathrm{C} \mathrm{NMR}: \quad\left(100 \mathrm{MHz}, \mathrm{CDCl}_{3}\right)$

176.57 (C(1)), 77.79 (C(9)), 60.52 (C(3)), 41.79 (C(2)), 41.19 (C(4)), 29.91 $(\mathrm{C}(7)), 27.13(\mathrm{C}(5,9)), 26.36(\mathrm{C}(6,8)), 26.32(\mathrm{C}(6,8)), 26.04(\mathrm{C}(5,9)), 14.91$ (C(12)), 14.19 (C(10)) 
IR: (neat)

3498 (w), 2979 (w), 2853 (s), 1721 (s), 1450 (m), 1376 (m), 1258 (m), 1179 (s), $1102(\mathrm{~m}), 1036(\mathrm{~m})$

MS: $\quad(\mathrm{EI}, 70 \mathrm{eV})$

$215\left(\mathrm{M}^{+}, 17\right), 197$ (37), 169 (9), 151 (28), 131 (100), 123 (26), 102 (83), 85 (64), $74(51), 57(34)$

Opt. Rot.: $\quad[\alpha]_{\mathrm{D}}^{24}+5.26(\mathrm{c}=1.0, \mathrm{EtOH})$

TLC: $\quad R_{f} 0.23$ (hexanes/EtOAc, 9/1) [UV(254)/ $\left.\mathrm{KMnO}_{4}\right]$

SFC: $\quad t_{R}(2 S, 3 S)-6 \mathbf{b j}, 8.30(67.8 \%) ; t_{R}(2 R, 3 R)-\mathbf{6 b j}, 9.16 \mathrm{~min}(32.8 \%)$ (Chiralpak OD, 150 bar, $40{ }^{\circ} \mathrm{C}, 3.0 \% \mathrm{CH}_{3} \mathrm{OH}$ in $\mathrm{CO}_{2}, 3.0 \mathrm{~mL} / \mathrm{min}, 220 \mathrm{~nm}$ )

Analysis: $\quad \mathrm{C}_{12} \mathrm{H}_{22} \mathrm{O}_{3}(214.16)$

Calcd: $\quad$ C, 67.26; $\quad$ H, $10.35 \%$

Found: $\quad$ C, $67.03 ; \quad H, 10.55 \%$

\section{Preparation of Methyl $(R)-3$-Trifluoromethylacetoxy-3-cyclohexylpropanoate $((R)-8)$}

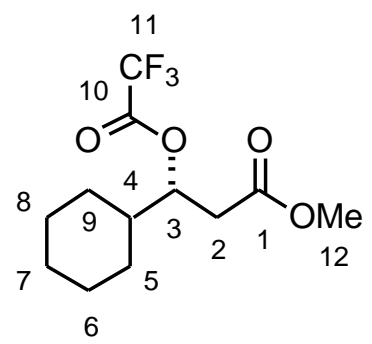

To a stirred solution of $61 \mathrm{mg}\left(1.1 \mathrm{mmol}, 1.0\right.$ equiv) of $(R)-\mathbf{4 j}$ in $\mathrm{CH}_{2} \mathrm{Cl}_{2}(1.0 \mathrm{~mL})$ was added $0.25 \mathrm{~mL}$ (1.76 mmol, 1.6 equiv) of trifluoroacetic anhydride at room temperature under nitrogen. After the addition was completed, the mixture was stirred for $12 \mathrm{~h}$. The reaction mixture was then concentrated in vacuo. Purification of the residue by silica gel chromatography (hexane/EtOAc, 10/1), afforded $88 \mathrm{mg}(95 \%)$ of $(R)-8$.

Data for $(R)-8$ :

${ }^{1} \mathrm{H}$ NMR: $\quad\left(400 \mathrm{MHz}, \mathrm{CDCl}_{3}\right)$

$5.33(\mathrm{dd}, J=6.6,6.1,1 \mathrm{H}, \mathrm{HC}(3)), 3.69$ (s, $3 \mathrm{H}, \mathrm{HC}(12)), 2.69$ (d, $J=6.8,2 \mathrm{H}, 2$ x HC(2)), 1.81-1.64 (m, 6 H, H(Cy)), 1.28-1.00 (m, 5 H, H(Cy)) 
${ }^{13} \mathrm{C} \mathrm{NMR}: \quad\left(100 \mathrm{MHz}, \mathrm{CDCl}_{3}\right)$

$170.56(\mathrm{C}(1)), 157.10(\mathrm{q}, J=41.9, \mathrm{C}(10)), 114.80(\mathrm{q}, J=286, \mathrm{C}(11)), 78.83$ (C(3)), 52.23 (C(12)), $41.27(\mathrm{C}(2)), 36.18$ (C(4)), 28.66 (C(7)), 27.87 (C(5)), $26.17(\mathrm{C}(9)), 25.93(\mathrm{C}(6)), 25.85(\mathrm{C}(8))$

IR: (neat)

2934 (s), 2858 (s), 1787 (s), 1745 (s), 1451 (m), 1439 (m), 1383 (m), 1339 (m), 1285 (m), 1262 (s), 1220 (s), 992 (m), 892 (w), 837 (w), 771 (m), 732 (m)

GC: $\quad t_{R}(S)-8,7.73(5.8 \%) ; t_{R}(R)-8,8.20 \mathrm{~min}(94.2 \%)\left(\mathrm{GTA}, 16 \mathrm{psi}, 100{ }^{\circ} \mathrm{C}\right.$ isothermal)

\section{Preparation of Ethyl (2S,3S)-3-Cyclohexyl-3-(3,5-dinitrobenzoyloxy)-2-methylpropanoate $((2 S, 3 S)-9)$}

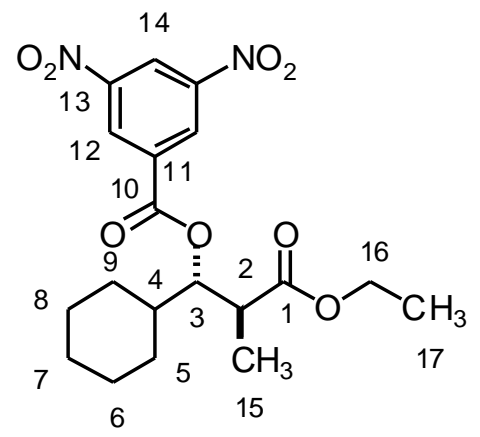

To a stirred solution of $134 \mathrm{mg}(0.627 \mathrm{mmol}, 1.0$ equiv $)$ of $(2 S, 3 S)-\mathbf{6 b j}$ in pyridine (1.0 $\mathrm{mL}$ ) and $1 \mathrm{~mL}$ of THF was added $145 \mathrm{mg}$ (0.627 mmol, 1.0 equiv) of 3,5 dinitrobenzoyl chloride at $0{ }^{\circ} \mathrm{C}$ under nitrogen. After the addition was completed, the mixture was stirred at room temperature for $5 \mathrm{~h}$. The reaction mixture was then diluted with $\mathrm{Et}_{2} \mathrm{O}(10 \mathrm{~mL})$, washed with 0.1 $\mathrm{M} \mathrm{HCl}(20 \mathrm{~mL})$, sat. $\mathrm{NaHCO}_{3}(20 \mathrm{~mL})$ and brine $(20 \mathrm{~mL})$. The organic layer was dried over $\mathrm{Na}_{2} \mathrm{SO}_{4}(2 \mathrm{~g})$ and filtered. The filtrate was concentrated in vacuo. Purification of the residue by silica gel chromatography (hexane/EtOAc, 9/1), afforded $220 \mathrm{mg}(86 \%)$ of $(2 S, 3 S)-9)$. 


\section{Data for $(2 S, 3 S)-\mathbf{9}$ :}

${ }^{1} \mathrm{H}$ NMR: $\quad\left(500 \mathrm{MHz}, \mathrm{CDCl}_{3}\right)$

9.22 (s, $1 \mathrm{H}, \mathrm{HC}(14)), 9.14$ (s, $2 \mathrm{H}, \mathrm{HC}(12)), 5.29$ (t, $J=6.10, \mathrm{HC}(3)), 4.12$ (q, $J$ $=7.08,2 \mathrm{H}, \mathrm{HC}(16)), 3.01$ (pent, $J=6.38,1 \mathrm{H}, \mathrm{HC}(2)), 1.83-1.65(\mathrm{~m}, 8 \mathrm{H}$, $\mathrm{HC}(5,6,8,9)), 1.27\left(\mathrm{t}, J=7.08,3 \mathrm{H}, \mathrm{H}_{3} \mathrm{C}(17)\right), 1.22\left(\mathrm{~d}, J=7.0,3 \mathrm{H}, \mathrm{H}_{3} \mathrm{C}(15)\right), 1.2-$ $0.9(\mathrm{~m}, 3 \mathrm{H}, \mathrm{HC}(4,7))$

${ }^{13} \mathrm{C} \mathrm{NMR}: \quad\left(125 \mathrm{MHz}, \mathrm{CDCl}_{3}\right)$ $173.60(\mathrm{C}(1)), 162.32(\mathrm{C}(10)), 148.94(\mathrm{C}(13)), 134.21$ (C(11)), $129.76(\mathrm{C}(12))$, 122.64 (C(14)), $81.64(\mathrm{C}(16)), 61.11(\mathrm{C}(3)), 41.53$ (C(2)), 39.39 (C(4)), 30.15 $(\mathrm{C}(7)), 27.44(\mathrm{C}(5,9)), 26.30(\mathrm{C}(6,8)), 26.22(\mathrm{C}(6,8)), 26.02(\mathrm{C}(5,9)), 14.43$ (C(17)), $14.33(\mathrm{C}(15))$

TLC: $\quad R_{f} 0.25$ (hexanes/EtOAc, 9/1) [UV(254)]

SFC: $\quad t_{R}(2 S, 3 S)-9,8.30(67.8 \%) ; t_{R}(2 R, 3 R)-9,9.16 \min$ (32.8\%) (Chiralpak OD, 150 bar, $40{ }^{\circ} \mathrm{C}, 3.0 \% \mathrm{CH}_{3} \mathrm{OH}$ in $\mathrm{CO}_{2}, 3.0 \mathrm{~mL} / \mathrm{min}, 220 \mathrm{~nm}$ )

\section{$\alpha$-Chloro(cyclohexylmethoxy)trichlorosilane (7)}

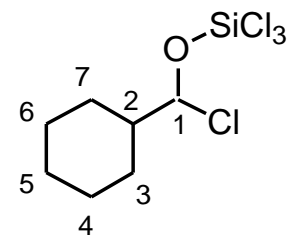

Into a dry NMR tube was added $30 \mu \mathrm{L}(0.25 \mathrm{mmol}, 1.0$ equiv $)$ of freshly distilled cyclohexanecarboxaldehyde and $1.0 \mathrm{~mL}$ of $\mathrm{CDCl}_{3}$ at room temperature under nitrogen. To this solution was added $2.2 \mu \mathrm{L}$ of ( $0.0125 \mathrm{mmol}, 0.05$ equiv) of HMPA followed by $34 \mu \mathrm{L}$ ( 0.3 mmol, 1.2 equiv) of $\mathrm{SiCl}_{4}$. The NMR tube was vortexed for 1 min to insure mixing. After 15 min the NMR tube was placed into the spectrometer at room temperature and a ${ }^{1} \mathrm{H}$ NMR spectrum was acquired. After $24 \mathrm{~h}$ at room temperature, $\mathrm{a}{ }^{13} \mathrm{C}$ NMR spectrum was acquired. Data for 7:

'H NMR: $\quad\left(500 \mathrm{MHz}, \mathrm{CDCl}_{3}\right)$

$5.78(\mathrm{~d}, J=4.88,1 \mathrm{H}, \mathrm{HC}(1)), 1.94-1.65$ (m, $6 \mathrm{H}, 6$ x H(Cy)), 1.30-1.10 (m, $5 \mathrm{H}$, 5 x H(Cy)) 
${ }^{13} \mathrm{C} \mathrm{NMR}: \quad\left(126 \mathrm{MHz}, \mathrm{CDCl}_{3}\right)$

92.95 (C(1)), 47.05 (C(2)), 28.03 (C(3)), 27.56 (C(7)), 26.19 (C(6)), 25.73 (C(4)), $25.63(\mathrm{C}(5))$

\section{References:}

(1) Denmark, S. E.; Wynn T. J. Am Chem. Soc. 2001, 123, 6199.

(2) Journet, M.; Cai, D.; DiMichele, L. M.; Larsen, R. D. Tetrahedron Lett. 1998, 39, 6427.

(3) Ireland, R. E.; Wipf, P.; Armstrong, J. D. J. Org. Chem. 1991 56, 650.

(4) Lutz, G. P.; Du, H.; Gallagher, D. J.; Beak, P. J. Org. Chem. 1996, 61, 4542.

(5) Carreira, E. M.; Singer R. A.; Lee, W. J. Am. Chem . Soc. 1994, 116, 8837.

(6) Denmark, S. E.; Winter, S. B. D.; Su, X.; Wong, K.-T. J. Am. Chem. Soc. 1996, 118, 7404.

(7) Fujitsawa, T.; Fujimura, A.; Sato, T. Bull. Chem. Soc. Jpn. 1988, 61, 1273.

(8) Wilcox, C. S.; Babston, R. E. J. Org. Chem. 1984, 49, 1451. 